\title{
A Reliable Leakage Reduction Technique for Approximate Full Adder with Reduced Ground Bounce Noise
}

\author{
Candy Goyal $\mathbb{C}^{1},{ }^{1}$ Jagpal Singh Ubhi, ${ }^{2}$ and Balwinder Raj ${ }^{3}$ \\ ${ }^{1}$ ECE Section, Yadavindra College of Engineering, Talwandi Sabo, Punjab, India \\ ${ }^{2}$ Department of ECE, SLIET, Longowal, Sangrur District, Punjab, India \\ ${ }^{3}$ Department of ECE, NIT Jalandhar, Punjab, India \\ Correspondence should be addressed to Candy Goyal; candygoyal@pbi.ac.in
}

Received 30 May 2018; Revised 30 September 2018; Accepted 15 October 2018; Published 16 December 2018

Academic Editor: Amir Sabbagh Molahosseini

Copyright (C) 2018 Candy Goyal et al. This is an open access article distributed under the Creative Commons Attribution License, which permits unrestricted use, distribution, and reproduction in any medium, provided the original work is properly cited.

In this paper, an effective and reliable sleep circuit is proposed, which not only reduces leakage power but also shows significant reduction in ground bounce noise (GBN) in approximate full adder (FA) circuits. Four 1-bit approximate FA circuits are modified using proposed sleep circuit which uses one NMOS and one PMOS transistor. The design metrics such as average power, delay, power delay product (PDP), leakage power, and GBN are compared with nine other 1-bit FA circuits reported till date. All the comparisons are done using post-layout netlist at $45 \mathrm{~nm}$ technology. The modified designs achieve reduction in leakage power and GBN up to $60 \%$ and $80 \%$, respectively, as compared to the best reported approximate FA circuits. The modified approximate FA also achieves $83 \%$ reduction in leakage power as compared to conventional FA. Finally, application level metrics such as peak signal to noise ratio (PSNR) are considered to measure the performance of all the proposed approximate FAs.

\section{Introduction}

In today's electronic driven epoch, demand of high speed electronic devices has put tremendous pressure on VLSI designers to develop low leakage and high speed arithmetic circuits. CMOS adders are having significant impact [1] on performance of overall system. Most of the advanced DSP algorithms also use addition as a primary operation, so any improvement in performance parameters of FA circuit can improve the whole system [2].

During the earlier phase of VLSI era, designers were focusing on miniaturization of MOS transistors to reduce chip size so as to achieve more and more portability for electronic devices. It is well known that if technology shrinks, threshold voltage and supply voltage also scale down to maintain the switching speed and performance, which results in exponential rise in leakage current [3] in nanoscale VLSI circuits. Leakage power has become critical issue[4], because electronic devices such as mobile phones, laptops, and other battery operated devices remain in standby mode for most of the time, which results in sharp battery discharge due to heavy leakage current [5]. Several techniques have been used in the past for reduction in leakage power in digital circuits but most commonly used technique is power gating [6]. It uses sleep transistors which provide high impedance between Vdd and GND during sleep mode to reduce leakage power. Although existing power gating techniques are very effective, all of them are having the disadvantage of large voltage fluctuations [7] during sleep to active mode of transition. During sleep period VGND node charges up to the supply voltage which creates instantaneous current peaks during mode transition. These voltage fluctuations are known as GBN as shown in Figure 1.

GBN was not the major problem in micrometer technologies but it is a serious concern at nanoscale VLSI design [8]. At deep submicron technology, intensity of GBN peaks is large enough to change the internal logic states of any circuit. The reliability of a circuit can be increased by curtailing its GBN.

During the last two decades various FA circuits were presented in literature for low power arithmetic circuits. However, a very few circuits are optimized for leakage power. Recently a sleep circuit with three NMOS transistors and one PMOS transistor is presented [9], which reduces leakage power and GBN but requires large silicon area. A circuit with 


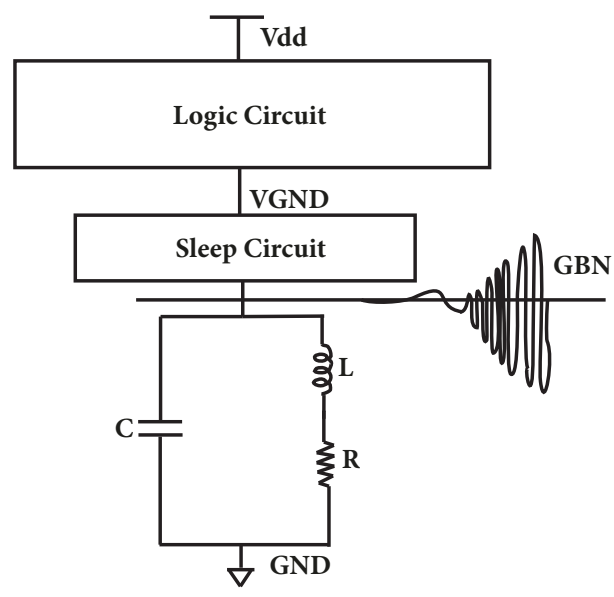

FIGURE 1: Ground bounce noise in conventional power gating circuits.

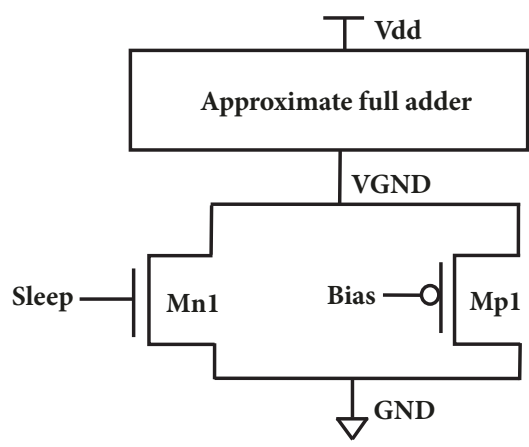

FIGURE 2: Block diagram of proposed sleep circuit with approximate FA.

tri-mode [10] is used to reduce leakage current and GBN which has a park mode between active and standby mode. It uses high Vth transistor along with the footer transistor. This technique has large propagation delay and it requires two separate control signals, which further increases complexity. Another circuit of power gating with stacking [11] is presented to reduce the leakage power and GBN in $10 \mathrm{~T}$ FA circuit. This technique has penalty of increase in delay and area due to extra transistors. Threshold voltage tuning [12] technique is presented recently. It uses one sleep transistor, a bias, and a parker transistor with forward biased body. Although there is decrease in GBN during sleep to active mode of transition, park control results in increase in delay due to twostep switching. Recently a new technique has been presented in literature [13] which uses two sleep transistors and one capacitor. In this approach one transistor gets the sleep signal immediately and the second transistor gets sleep signal after some delay. Two GBN peaks and need for extra buffers are the limitations of this approach. Recently, approximation [1417] is used as one of the possible solutions for low power VLSI design. In the past, several circuit styles of approximate adders are presented to reduce the average power and delay of the circuit, but to the best of our knowledge, leakage power is not improved. Two imprecise FA circuits [18] are presented, which have faster and lower power operation. They show reduction in dynamic power dissipation and transistor count as compared to conventional adder but leakage power is not considered for optimization. Four approximate [19] one-bit FAs are presented by logic reduction at transistor level. Average power dissipation and number of transistors are reduced at the expense of accuracy in conventional adder, but they consume large leakage power. Another inexact FA [20] is presented which used 10 transistors for one-bit FA. Although it improves dynamic power, circuit does not have full voltage swing for sum signal which reduces the noise margin and hence is not suitable for large cascaded arithmetic circuits. Leakage power is also not reported in this paper which might be high due to the two pass transistors used

Although leakage power and GBN have been reduced, further reduction in peaks of GBN is still possible. Most of the existing approximate adders consume large leakage power, which makes them unsuitable for long chain arithmetic circuits. In this article, we have modified the approximate adders with proposed sleep circuit to reduce leakage power and GBN.

\section{Proposed Circuit}

The modified structure of approximate adder is shown in Figure 2 with proposed sleep circuit between VGND and GND terminal.

Four approximate FAs [19] are tested with proposed sleep circuit. These modified approximate adders are named as MAA1, MAA2, MAA3, and MAA4 in this paper as shown in Figures 3, 4, 5, and 6, respectively, where MAA stands for modified approximate adder.

We have calculated the $\mathrm{W} / \mathrm{L}$ ratio of all the modified as well as existing approximate FAs according to the basic principle of CMOS technology in which effective width of pull-up network is double the effective width of pull-down network. It gives the equal rise and fall times of all the output signals. The transistor sizes of all the modified approximate FA cells are included in Figures 3-6. Length of all the transistors are kept at $45 \mathrm{~nm}$ which is the minimum length at $45 \mathrm{~nm}$ technology.

Circuit diagram of proposed sleep circuit is shown in Figure 7. The sleep circuit has three modes of operation:

(i) active mode,

(ii) sleep mode,

(iii) sleep to active mode transition.

All the simulations are performed using 45nm technology having threshold voltage of NMOS and PMOS transistor given as follows.

$$
\begin{aligned}
& \mathrm{V}_{\text {th }}(\mathrm{NMOS})=0.62 \mathrm{~V} \\
& \mathrm{~V}_{\text {th }}(\mathrm{PMOS})=-0.58 \mathrm{~V}
\end{aligned}
$$




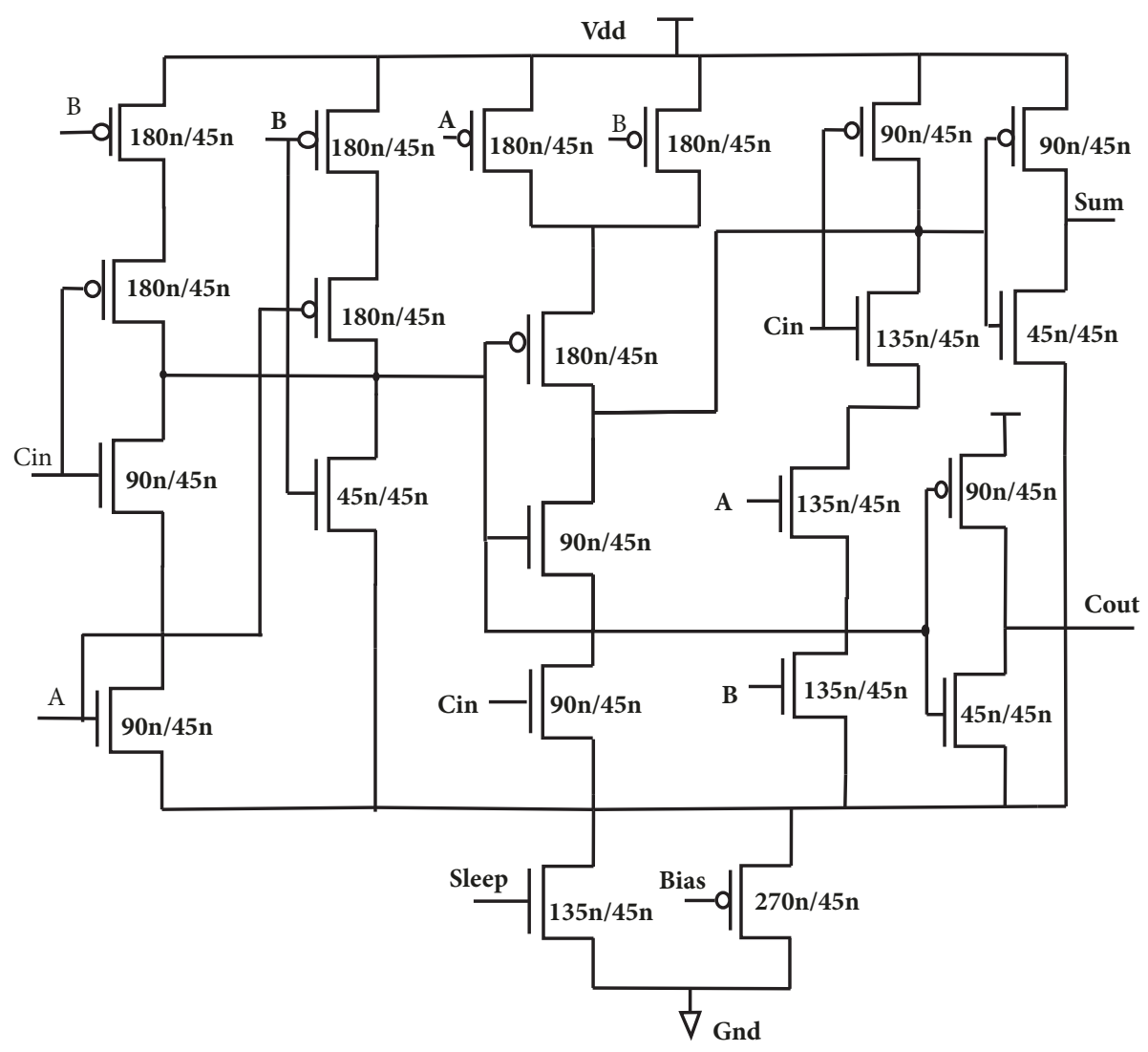

Figure 3: Modified approximate adder-1 (MAA1).

2.1. Active Mode. In active mode, sleep signal will be at logic high. It will switch NMOS transistor of the sleep circuit to ON state and VGND terminal will connect to real GND which forces PMOS transistor into cutoff state. In active mode, NMOS transistor offers very low resistance and PMOS transistor offers very high resistance. Equivalent circuit of both the sleep transistors in active mode is shown in Figure 8, where $C_{D S N}$ is capacitance between drain and source of NMOS transistor, $\mathrm{C}_{\mathrm{SDP}}$ is the capacitance between source and drain of PMOS transistor, and Rnon is the resistance between drain and source of NMOS transistor.

In active mode output of logic circuit will be the same as it was in normal circuit.

2.2. Sleep Mode. In sleep mode, sleep signal will gradually fall from Vdd to $0 \mathrm{~V}$ and potential at VGND node will increase gradually as shown in Figure 9.

As long as the voltage level of sleep signal is higher than the threshold voltage of Mn1 transistor, Mnl will operate in saturation region and potential at VGND node will be very small which is shown during time T1 in Figure 9. During time T2 sleep signal reaches $0 \mathrm{~V}$ and $\mathrm{Mn} 1$ will go in cutoff state. Potential rises sharply at VGND node during time T2 and when it increases beyond $0.5 \mathrm{~V}, \mathrm{Mpl}$ will turn ON. As the bias at gate of $\mathrm{Mp} 1$ is $-0.1 \mathrm{~V}, \mathrm{~V}_{\mathrm{gs}}$ of $\mathrm{Mpl}$ at this time will be given as follows.

$$
\mathrm{V}_{\mathrm{gs}}=\mathrm{V}_{\mathrm{g}}-\mathrm{V}_{\mathrm{s}}=-0.1 \mathrm{~V}-0.5 \mathrm{~V}=-0.6 \mathrm{~V}
$$

As threshold voltage of PMOS transistor is $-0.58 \mathrm{~V}, \mathrm{Mp} 1$ transistor will turn $\mathrm{ON}$ during time T3. The ON time of Mp1 transistor during sleep period will depend upon the value of bias voltage. The higher the value of bias voltage is, the less the turn $\mathrm{ON}$ time will be. The equivalent circuit of sleep circuit at this condition is shown in Figure 10.

During time T3 Mp1 will discharge the potential from VGND node to GND; as it goes slightly below $0.5 \mathrm{~V}, \mathrm{Mp} 1$ transistor goes in cutoff mode because $V_{\text {gs }}$ will not be sufficient to turn $\mathrm{ON}$ the Mp1 transistor. Therefore, both transistors $\mathrm{Mnl}$ and $\mathrm{Mpl}$ will be in cutoff state simultaneously after time T3 which is the stable state of the circuit. Equivalent circuit of sleep circuit at this condition is shown in Figure 11.

Potential at various nodes during sleep mode are shown in Figure 12.

2.3. Sleep to Active Mode Transition. In sleep to active mode transition, sleep signal changes from $0 \mathrm{~V}$ to Vdd and potential at gate of NMOS transistor starts rising. Whenever the potential at the gate of NMOS transistor increases beyond the threshold voltage, it gets turned ON. NMOS transistor will turn $\mathrm{ON}$ in linear region because potential at VGND node (in Figure 12) in sleep mode ensures to turn ON Mnl in linear mode. Thus, it greatly reduces the peaks of GBN during the switching from sleep to active mode of the circuit. 


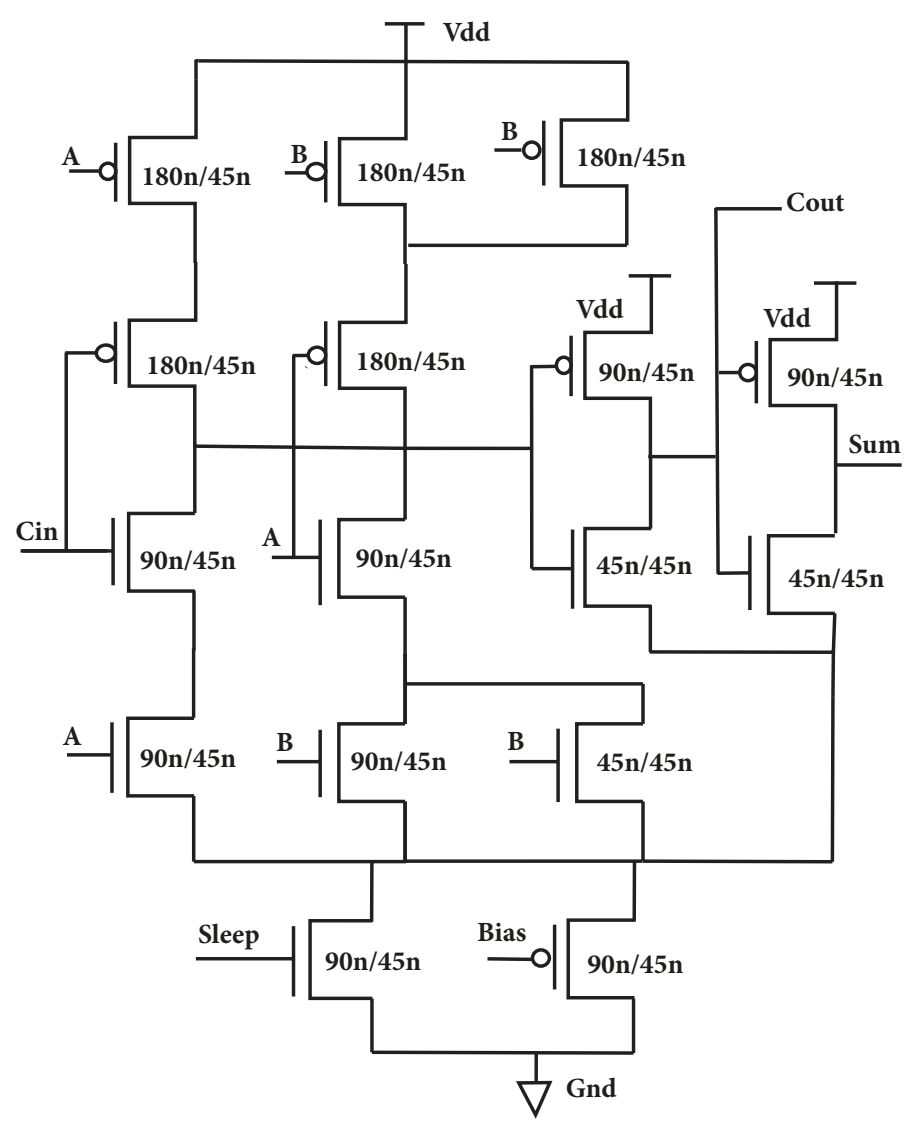

FIgURE 4: Modified approximate adder-2 (MAA2).

\section{Simulation Setup}

To demonstrate the performance of our modified approximate FA, post-layout simulation results are extracted at $45 \mathrm{~nm}$ technology. All the circuits, existing as well as modified, are tested under the same environment conditions. Simulation setup is shown in Figure 13. A load of $0.8 \mathrm{fF}$ is connected at the output of each approximate FA which is equivalent load of four minimum unit inverters at $45 \mathrm{~nm}$ technology.

\section{Results and Discussion}

The results are discussed in this section with respect to the average power, delay, PDP, leakage power, and GBN performance metrics. The extraction of all the performance metrics for existing and modified adders has been done through post-layout simulations. The modified design has the following advantages as compared to the existing sleep circuit [11].

(i) The proposed sleep circuit has very simple circuitry which used only one NMOS and one PMOS transistor with normal $\mathrm{V}_{\text {th }}$.

(ii) PMOS transistor is used with negative gate bias. This maintains the potential in such a way so that NMOS transistor of sleep circuit will turn ON in linear region during the sleep to active mode transition which reduces the GBN as compared to the existing sleep circuit [11].

(iii) The proposed sleep circuit has symmetric and compact layout as compared to the existing sleep circuit [11].

(iv) Simulation results at various temperature and voltage values confirm the stability of the modified circuit.

4.1. Layout of Modified Design. Layouts of all the existing as well as modified FAs under consideration in this paper are designed using Cadence Virtuoso layout editor at $45 \mathrm{~nm}$ technology. These layouts are used to perform physical verification (DRC, LVS) and extraction of post-layout netlist. Post-layout netlists are used for simulation and extraction of performance parameters of all the FAs in this paper. Layouts of modified approximate FAs are shown in Figures 14-17, respectively.

4.2. Average Power and Delay. To extract the average power and delay of all the FAs under consideration, standard test input patterns [21] have been used, which are shown in Figures 18 and 19, respectively. These patterns cover all the possible worst case switching nodes and give very accurate results. In this paper, maximum frequency of input data is $500 \mathrm{MHz}$. The output waveforms of all the modified approximate FAs in active mode are shown in Figure 18. 


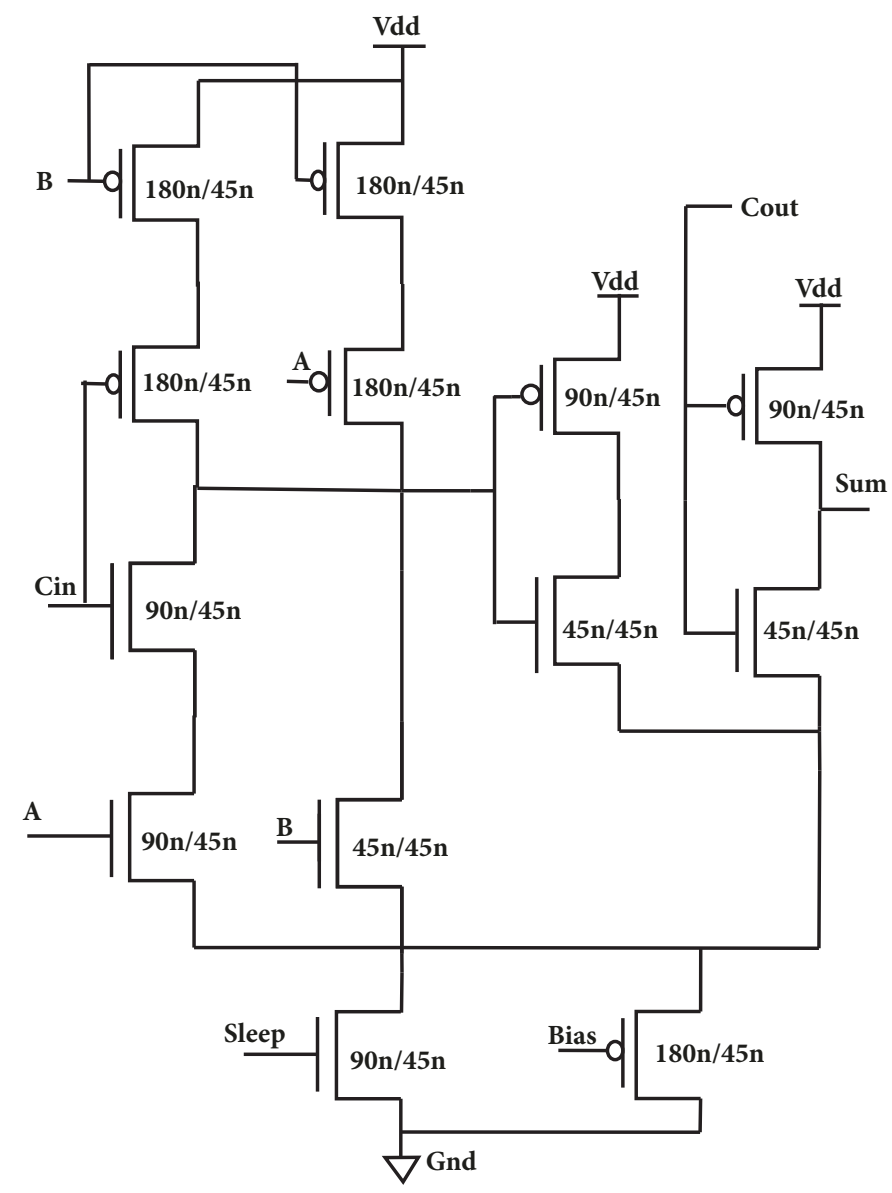

FIGURE 5: Modified approximate adder-3 (MAA3).

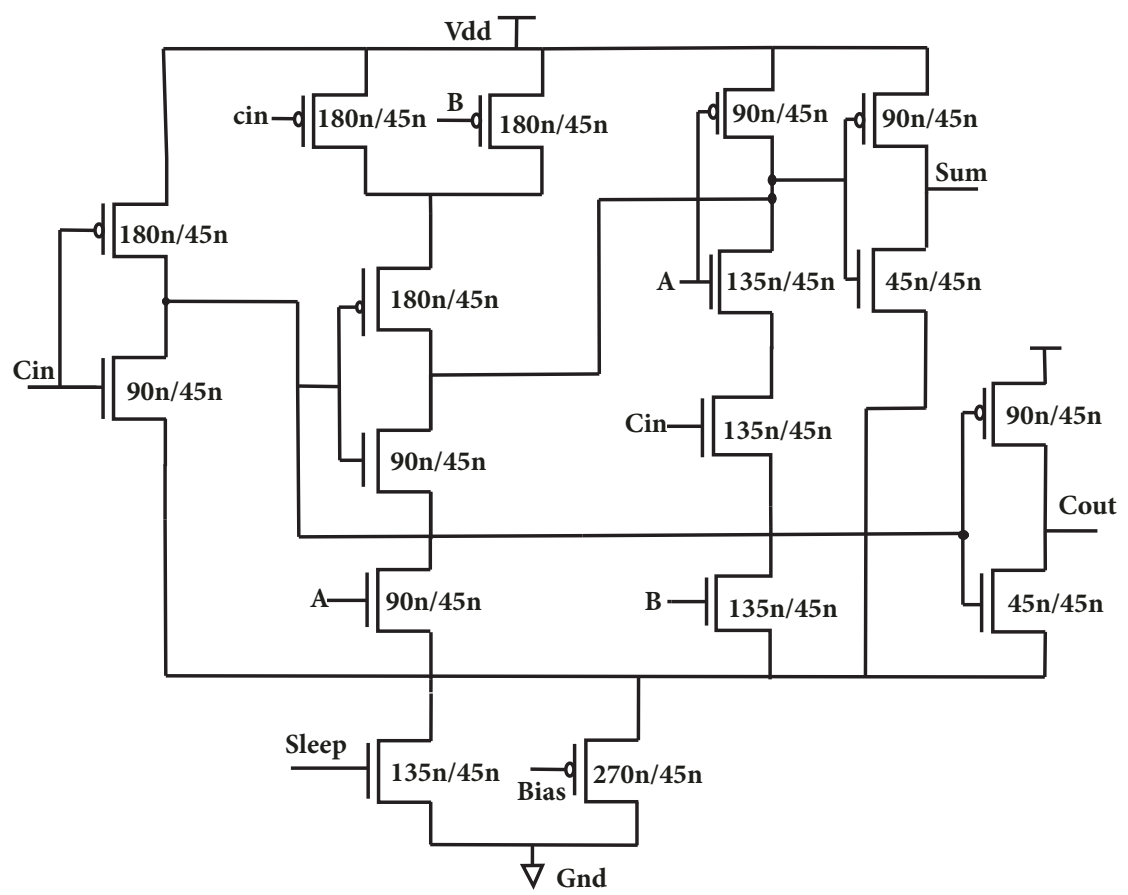

FigurE 6: Modified approximate adder-4 (MAA4). 


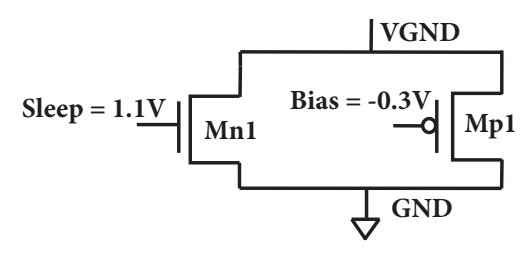

Figure 7: Circuit of proposed sleep circuit in active mode.

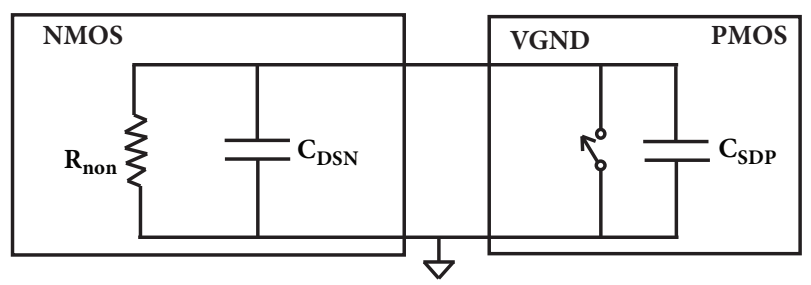

FIGURE 8: Equivalent circuit of modified sleep circuit in active mode.

Average power includes three types of power dissipation in the CMOS circuit. Mathematically it is given as follows.

$$
\mathrm{P}_{\text {average }}=\mathrm{P}_{\text {switching }}+\mathrm{P}_{\text {static }}+\mathrm{P}_{\text {short-circuit }}
$$

Switching power dissipation is the average power dissipation due to the switching of all the nodes in the circuit during working condition. Static power is the power dissipated by the circuit during idle state of the circuit. Short-circuit power is the power dissipation due to the simultaneous conduction of pull-down and pull-up network connecting between the Vdd and GND of the circuit.

The propagation delay is calculated when a $50 \%$ change in input signal from 0 to 1 or 1 to 0 corresponds to $50 \%$ change in output signals from either 0 to 1 or 1 to 0 transition. In this paper, the input pattern as shown in Figure 19 is used for measuring the delay. It has total 56 input patterns that cover maximum possible input/output transitions for all the possible input combinations of FA.

4.3. Leakage Power. The leakage power is measured in idle mode of the circuit. Basically leakage current is a combination of subthreshold and gate oxide leakage current. Mathematically, leakage current is given as

$$
\mathrm{I}_{\text {leakage }}=\mathrm{I}_{\text {subthreshold }}+\mathrm{I}_{\text {gateleakage }}
$$

where

$\mathrm{I}_{\text {leakage }}$ is the total leakage current of circuit,

$\mathrm{I}_{\text {subthreshold }}$ is the subthreshold leakage current,

$I_{\text {gateleakage }}$ is the gate leakage current.

$$
I_{\text {subthreshold }}=I_{0} e^{\left(V_{g s}-V_{t h}\right) / \eta V_{T}}\left(1-e^{-V_{d s} / V_{t h}}\right)
$$

where

$$
I_{0}=\mu_{0} C_{o x}\left(\frac{W_{e f f}}{L_{e f f}}\right) V_{T}^{2} e^{1.8}
$$

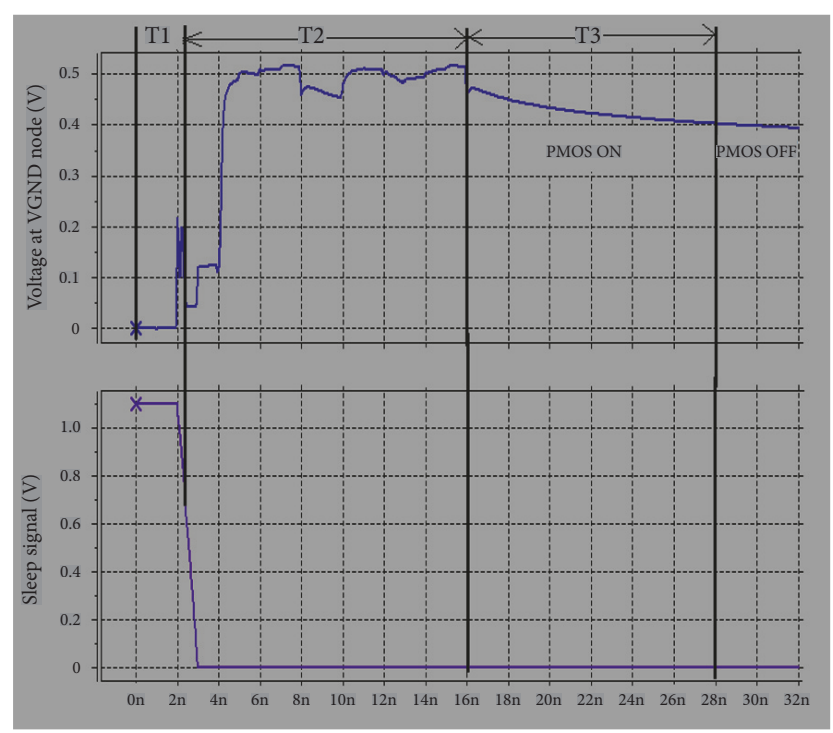

FIGURE 9: Potential at VGND node during sleep signal changes from Vdd to $0 \mathrm{~V}$.

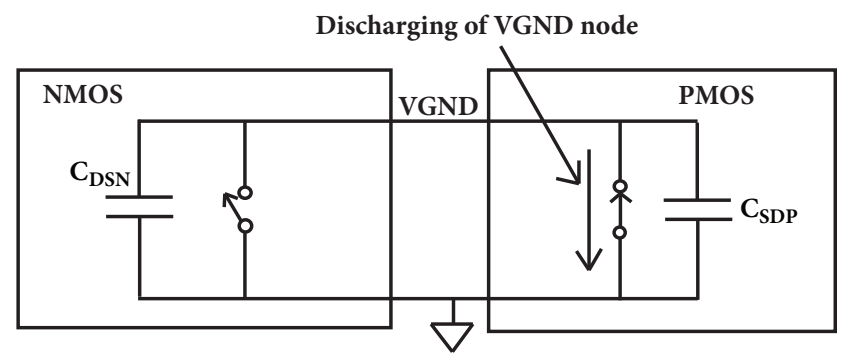

FIGURE 10: Equivalent circuit diagram of proposed sleep circuit in sleep mode (when PMOS transistor turns ON).

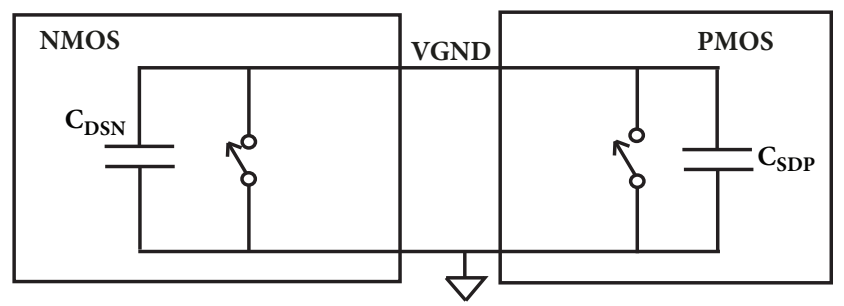

FIGURE 11: Equivalent circuit of proposed sleep circuit in sleep mode (when sleep signal is at logic low).

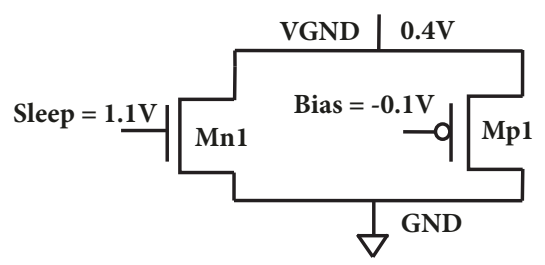

Figure 12: Proposed sleep circuit in sleep mode. 


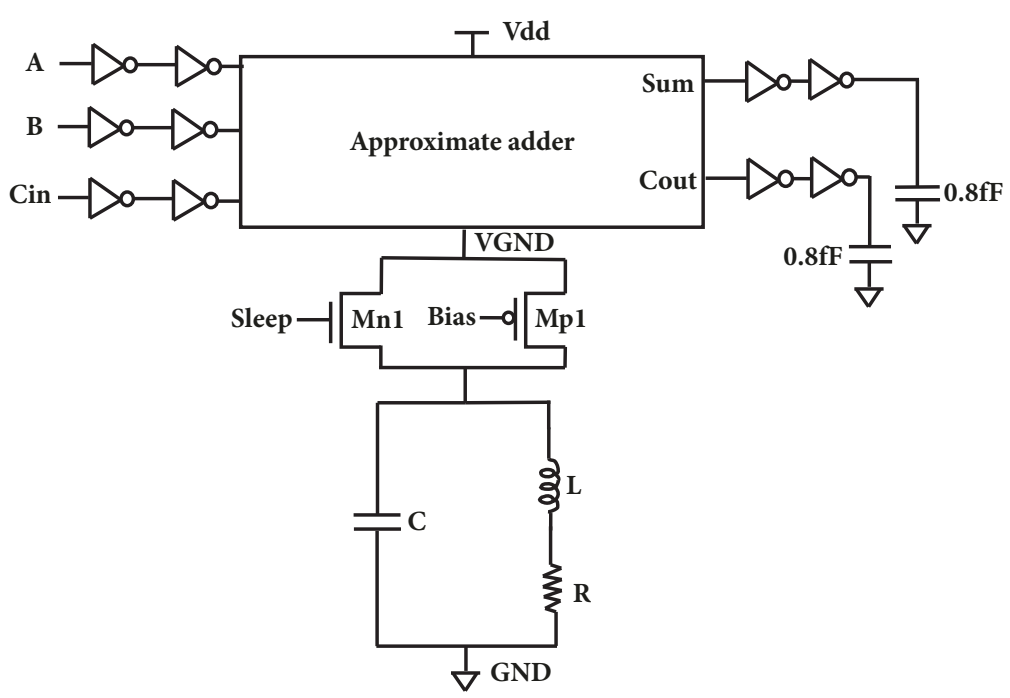

FIGURE 13: Simulation setup for proposed and existing approximate FA.

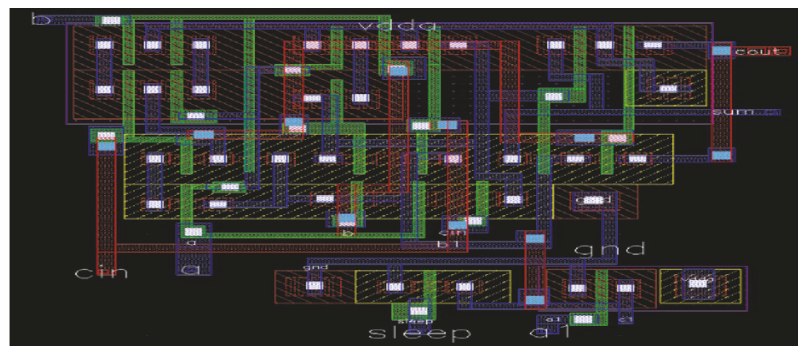

FIgURE 14: Layout of MMA1 adder.

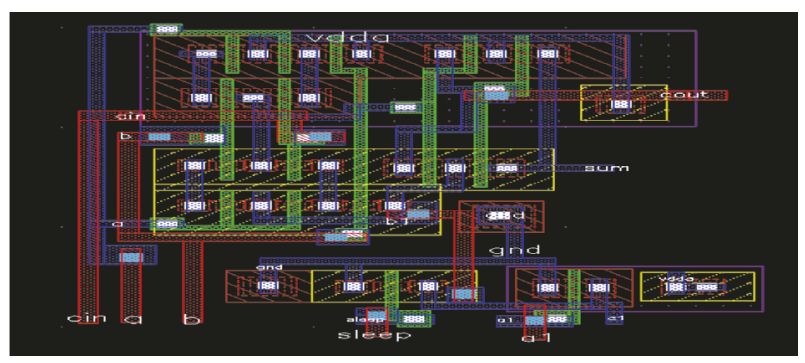

FIgURE 15: Layout of MMA2 adder.

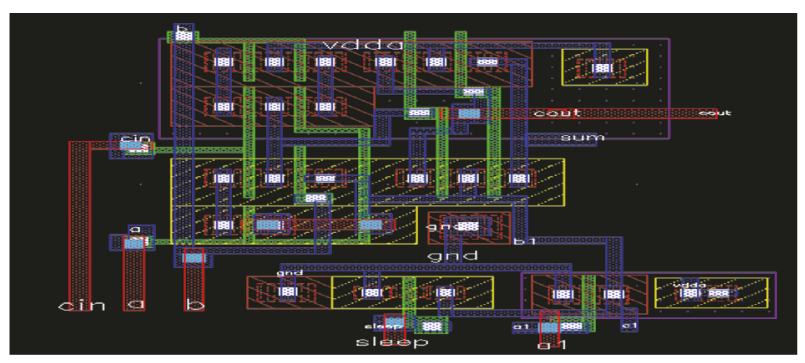

FIgURE 16: Layout of MMA3 adder.

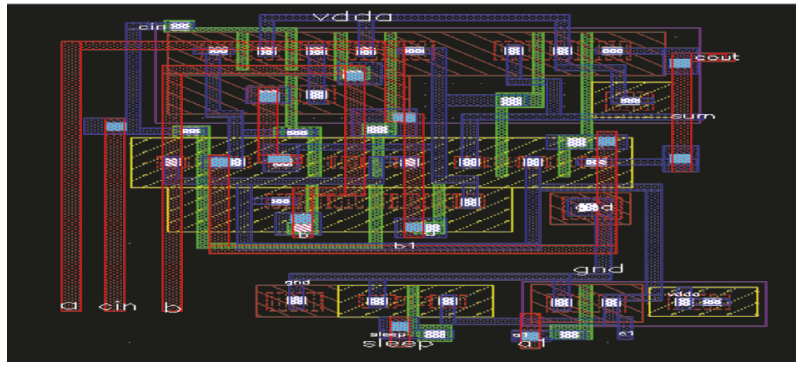

FIgURE 17: Layout of MMA4 adder.

where

$\mu_{0}$ is the mobility of the MOS transistor,

$\mathrm{C}_{\text {ox }}$ is the capacitance of gate oxide,

$\mathrm{W}_{\text {eff }}$ is the effective width of MOS transistor,

$L_{\text {eff }}$ is the effective length of MOS transistor,

$\mathrm{V}_{\mathrm{T}}$ is the volt equivalent of temperature,

$\eta$ is the drain induced barrier lowering coefficient,

$\mathrm{V}_{\mathrm{gs}}$ is the gate to source voltage of MOS transistor,

$\mathrm{V}_{\text {th }}$ is the threshold voltage of MOS transistor,

$\mathrm{V}_{\mathrm{ds}}$ is the drain to source voltage of MOS transistor.

Similarly gate oxide leakage current is given as

$$
I_{\text {gateleaakge }}=K_{2} W\left(\frac{V}{T_{o x}}\right)^{2} e^{-\propto T_{o x} / V_{T}}
$$

where

$\mathrm{T}_{\mathrm{ox}}$ is the gate oxide thickness,

$\mathrm{V}_{\mathrm{T}}$ is the thermal voltage, 


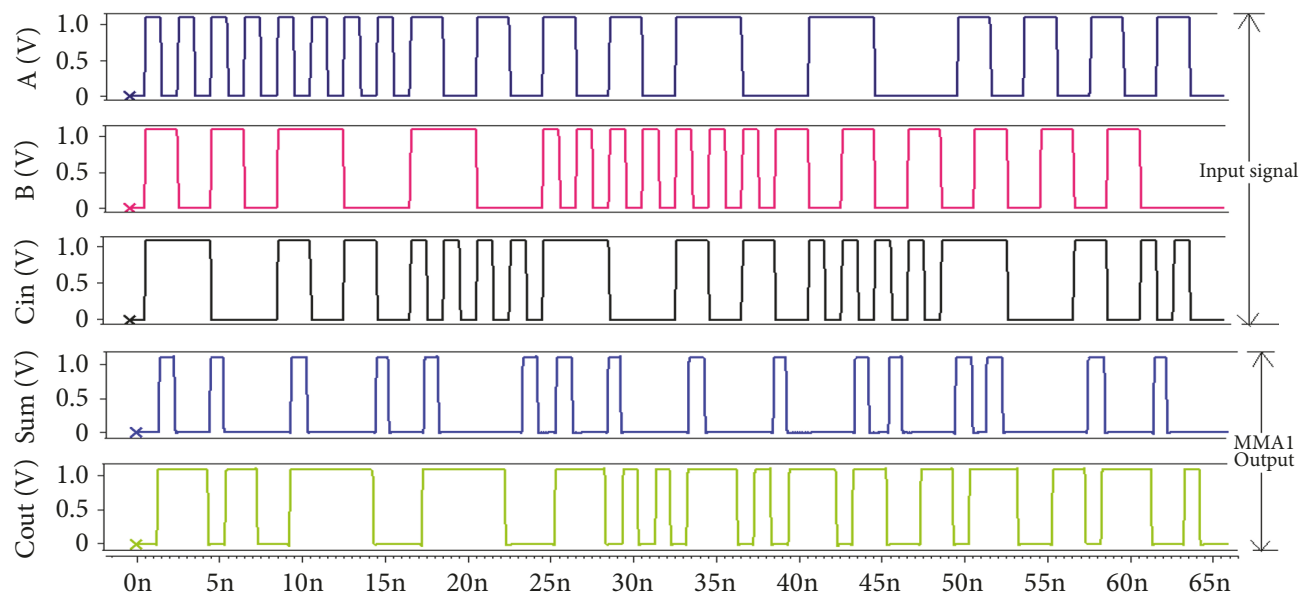

(a)

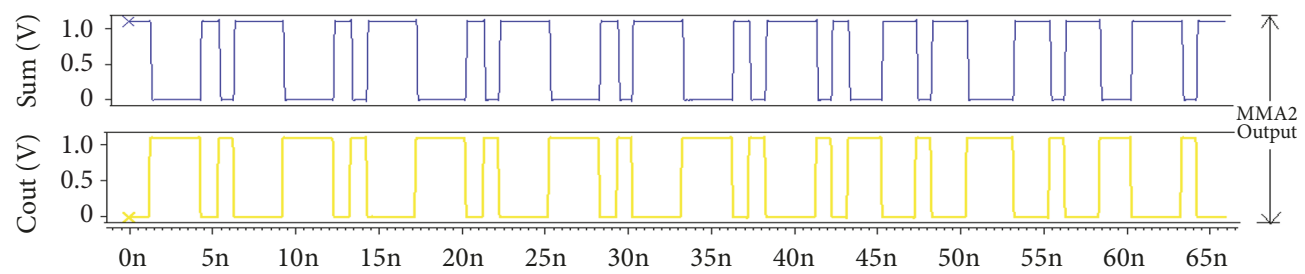

(b)

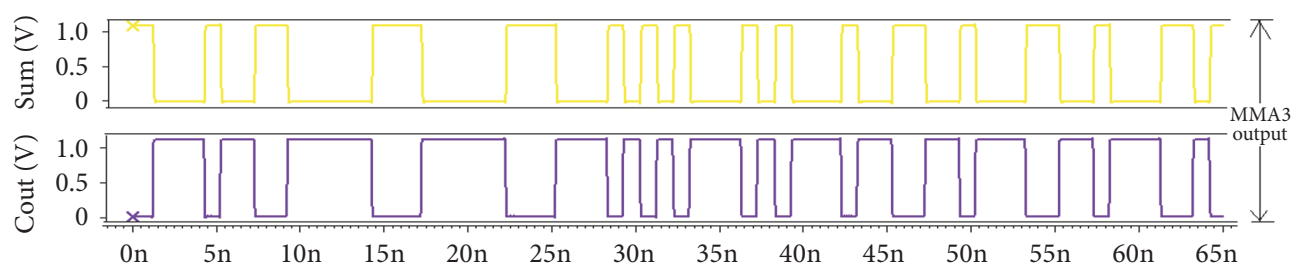

(c)

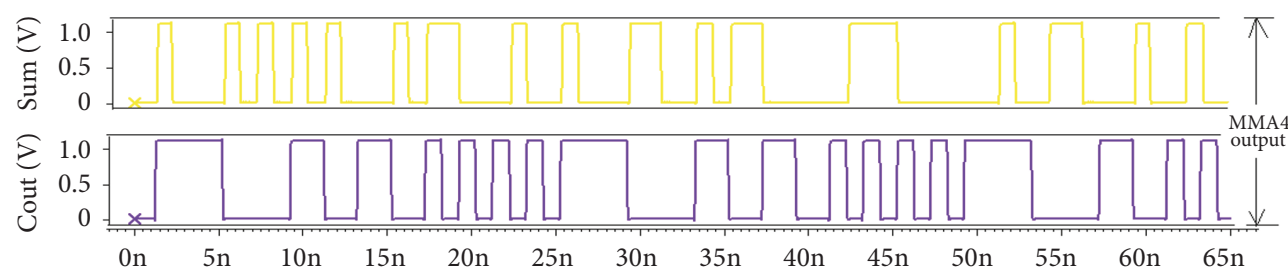

(d)

FIGURE 18: (a) Stimulus for average power dissipation for proposed and existing FAs. (a) Output waveforms of MMA1 adder. (b) Output waveforms of MAA2 adder. (c) Output waveforms of MAA3 adder. (d) Output waveforms of MAA4 adder.

$\mathrm{W}$ is the width of transistor,

$\mathrm{K}_{2}$ and $\alpha$ are experimentally derived parameters.

At $65 \mathrm{~nm}$ technology subthreshold leakage is dominant. As the technology is shrinking below $65 \mathrm{~nm}$, gate leakage current is becoming dominant factor as compared to subthreshold leakage current. During the stationary state of the circuit, data will not change at the input of the circuit; there will be stable data at the input. In FA, there are 3 inputs, so $2^{3}=8$ possible data combinations. Out of 8 possible combinations any combination can exist during idle state; leakage power is strongly dependent on data present at inputs during idle state. To measure accurate leakage power we have measured the leakage power for each possible data input and the average of all such values is the final leakage power of the circuit. The same procedure was adopted to measure the leakage power for all the FA circuits under consideration. Table 1 shows the leakage power values for all the existing and modified FA circuits.

4.4. GBN Calculations. GBN is the voltage fluctuations during sleep to active mode transition of circuit which is also known as simultaneous switching. GBN is a serious issue in deep submicron technology. In this paper, the behavior of GBN peaks is examined for existing and modified FAs under consideration. To measure the GBN, we have used the 


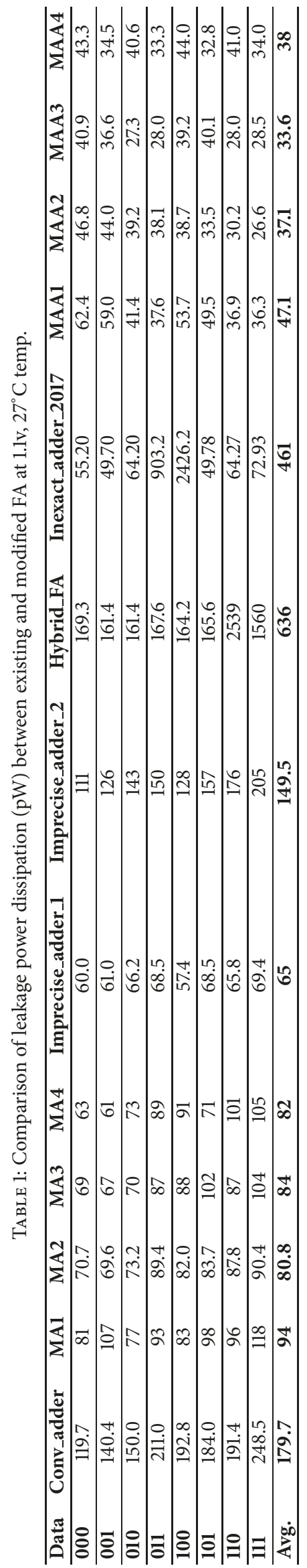



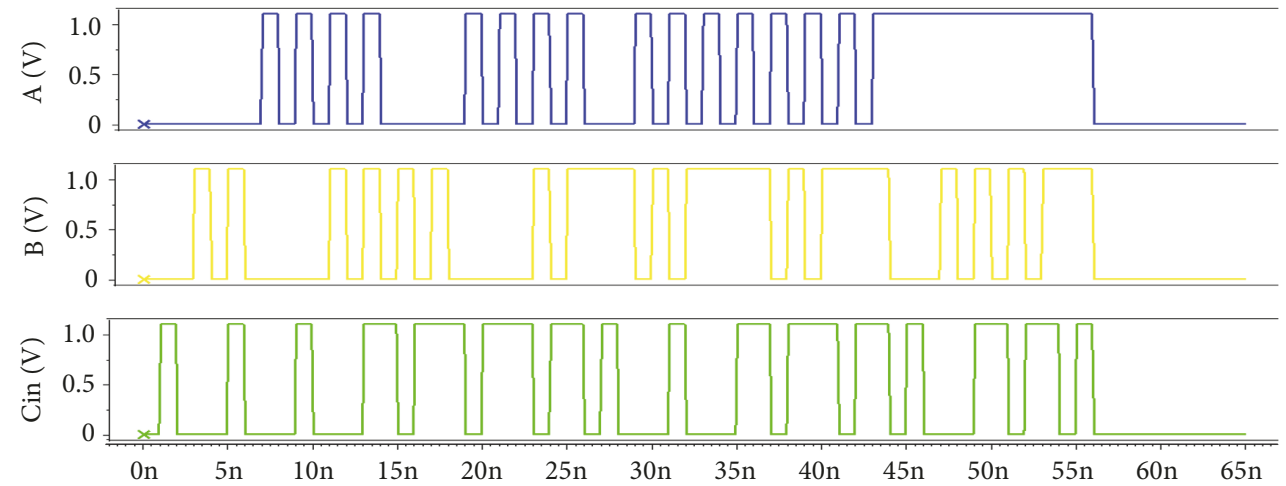

FIGURE 19: Input stimuli used to calculate delay for existing and modified approximate FA under consideration.

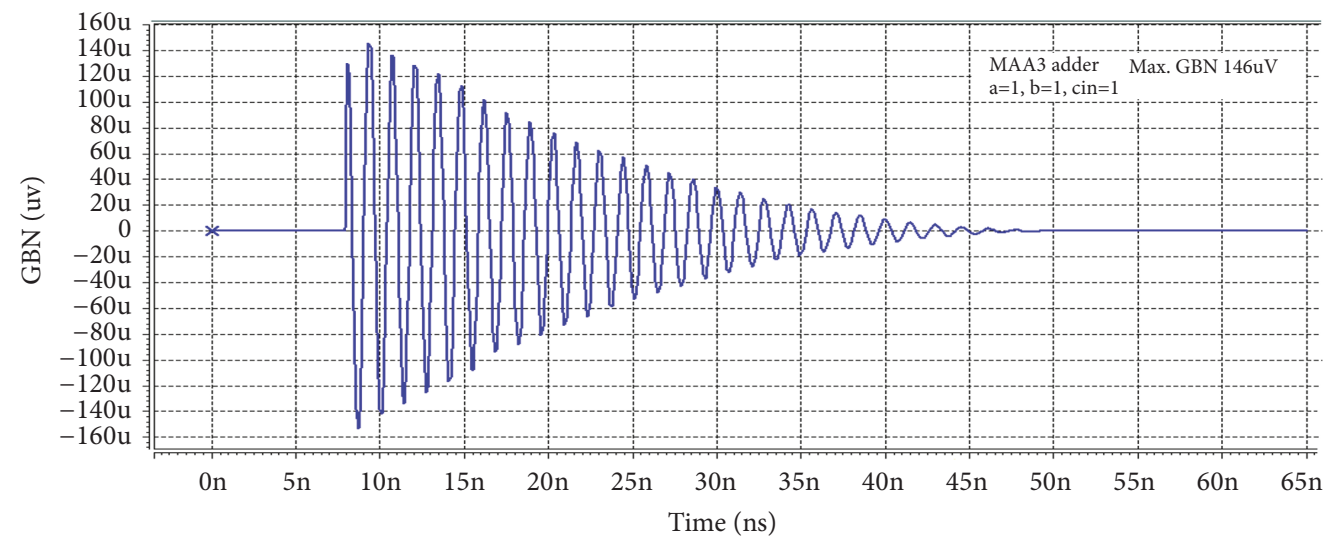

FIGURE 20: GBN peaks of modified approximate FA (MAA3).

electrical characteristics of DIP-40 package model [22] which has parasitic resistance $R$, inductance $L$, and capacitance $C$ on the GND line as shown in Figure 13. The standard values of parasitic elements $\mathrm{R}=217 \mathrm{~m} \Omega, \mathrm{L}=8.18 \mathrm{nH}$, and $\mathrm{C}=5.32 \mathrm{pF}$ are used to measure GBN. GBN peaks for all the existing and modified adders are obtained through these parameter values. As GBN is measure during transition of sleep to active mode and in active mode, we have selected bias voltage as $-0.3 \mathrm{~V}$, so all the GBN measurements are done at $-0.3 \mathrm{~V}$ bias value. Further, GBN depends on the input data values during idle state of the circuit because different input data produces different voltage fluctuations at VGND node. We have measured the GBN for all the possible input data values and maximum value of GBN peak is shown in Table 2. Result shows that MAA3 has the lowest GBN peaks. The waveforms of GBN for MAA3 modified as well as existing (MA3) are shown in Figures 20 and 21, respectively.

In deep submicron technology, temperature and voltage variations can significantly affect the performance of any digital circuit. It is because environment parameter variations are posing greater challenges in the design of scaled CMOS technologies. Hence, the effect of temperature and voltage variations on the leakage power, GBN, and PDP of modified as well as existing approximate FAs is studied, which is shown in Figures 22-28.
Variations of leakage power with respect to temperature are shown in Figure 23. It is clear from Figures 23 and 24 that leakage power increases with the increase in voltage and temperature. Because leakage power is directly proportional to supply voltage and increase in temperature, thermal vibrations of charge carriers increase and hence the leakage current. Variations are least in all the modified approximate FAs as compared to existing FAs.

Effect of GBN with temperature and voltage variations is shown in Figures 24 and 25, respectively. GBN variations are very serious issue at nanoscale VLSI design. As the temperature increases, the saturation current of sleep transistor decreases due to the reduction in carrier mobility which further reduces the peak amplitude of the GBN, so GBN reduces as the temperature rises, which is shown in Figure 24. Variations in temperature result in voltage fluctuations at VGND node. Voltage fluctuations should be as small as possible for reliable circuit. In our investigation we have found that MMA3 has the least variations in GBN with temperature and hence achieved high reliability and stability. Effect of voltage variations on GBN is shown in Figure 25. The peak of GBN is directly proportional to supply voltage. If we increase the supply voltage of the circuit, then voltage fluctuation on VGND node also increases and hence the GBN increases. 


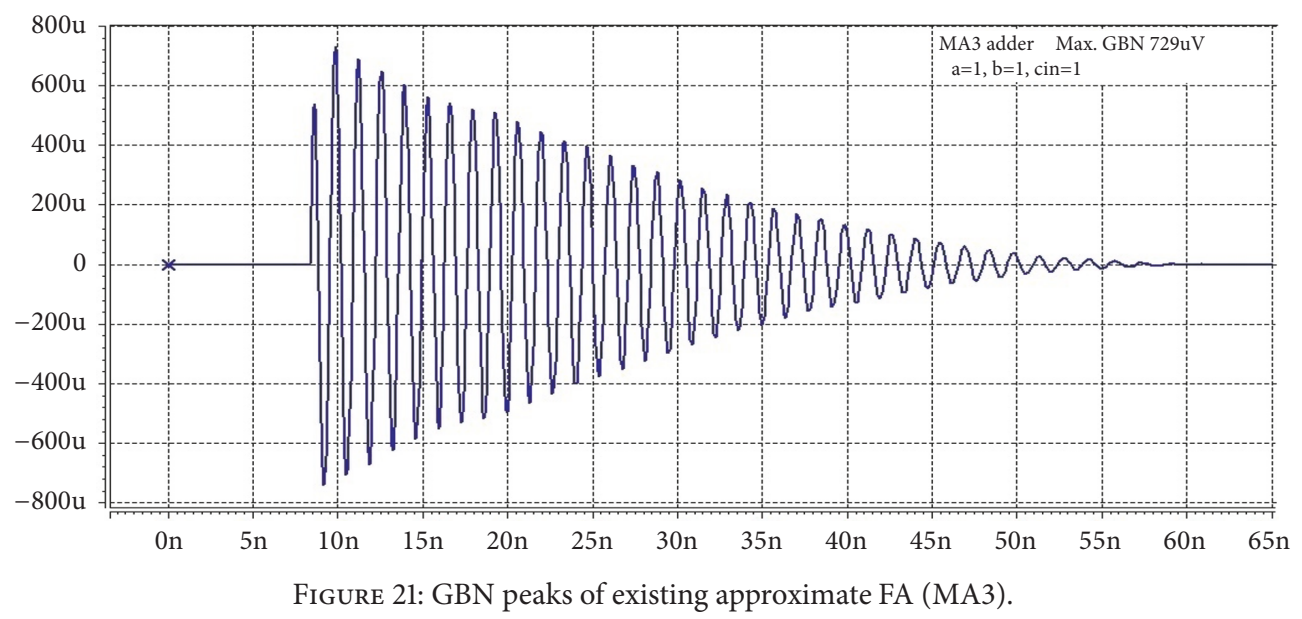

TABLE 2: Comparison of post layout simulation results between existing and modified $\mathrm{FA}$ at $1.1 \mathrm{~V}, 27^{\circ} \mathrm{C}$ temp.

\begin{tabular}{|c|c|c|c|c|c|}
\hline Scheme & Average Power $(\mu \mathrm{W})$ & Delay (pS) & PDP (aJ) & Leakage power(pW) & $\mathrm{GBN}(\mu \mathrm{V})$ \\
\hline \multicolumn{6}{|l|}{ Existing FA circuit } \\
\hline Conv_adder & 12.2 & 322 & 3928 & 180 & 890 \\
\hline MA1 [19] & 10 & 398 & 3980 & 94 & 856 \\
\hline MA2 [19] & 8.1 & 368 & 2980 & 81 & 791 \\
\hline MA3 [19] & 7.8 & 277 & 2160 & 84 & 753 \\
\hline MA4 [19] & 9.2 & 362 & 3330 & 82 & 808 \\
\hline Imprecise_adder_1 [18] & 7.7 & 361 & 2780 & 65 & 732 \\
\hline Imprecise adder_2[18] & 11.8 & 360 & 4248 & 149 & 879 \\
\hline Inexact_adder_2017 [20] & 7.5 & 361 & 2707 & 461 & 541 \\
\hline FA (hybrid_pass_logic) [21] & 11 & 879 & 9669 & 636 & 587 \\
\hline \multicolumn{6}{|l|}{ Modified approximate FA } \\
\hline MMA1 & 9.8 & 479 & 4694 & 47 & 191 \\
\hline MMA2 & 8.0 & 408 & 3264 & 37 & 146 \\
\hline MMA3 & 7.7 & 316 & 2433 & 34 & 145 \\
\hline MMA4 & 9.1 & 325 & 2957 & 38 & 205 \\
\hline
\end{tabular}

Figure 26 shows the effect of voltage variations on PDP. As the voltage increases, PDP decreases, because delay decreases more sharply as compared to the increase in average power dissipation with respect to increase in voltage.

The PDP increases for all the adders as the temperature rises as shown in Figure 27, because of increase in average power dissipation and delay with respect to temperature, and hence the PDP increases.

To evaluate the circuit performance more precisely we have studied the effect of output load variations with respect to PDP for all the existing as well as proposed designs under consideration in this paper. It is clear from Figure 28 that as the output load increases, PDP increases. It is because delay and dynamic power both are directly proportional to capacitance which results in increases in PDP with the increase in output load.

4.5. Motion Detection Using Proposed Approximate FAs. In order to measure the application level performance of proposed approximate FA, we have used a practical image processing application of motion detector.

Figures 29(a) and 29(b) show two images with slight differences which we have used to detect the movement of the object. These two images are converted into binary matrices named as $n_{1}$ and $n_{2}$, respectively, using MATLAB. Then they subtracted using

$$
Q(i, j)=n_{1}(i, j)-n_{2}(i, j)
$$

where $i$ and $j$ are the rows and columns of matrices.

$i$ is the number of rows in binary matrices.

$\mathrm{j}$ is the number of columns in binary matrices.

These images are converted into binary matrices using MAT$\mathrm{LAB}$ program. $\mathrm{Q}(\mathrm{i}, \mathrm{j})$ matrices will determine the possible movement in the two picture frames. If there is no movement, then the two images will be exactly the same and their resultant matrices will be completely zero. If there is a movement, then corresponding shadows will exist in the resultant 


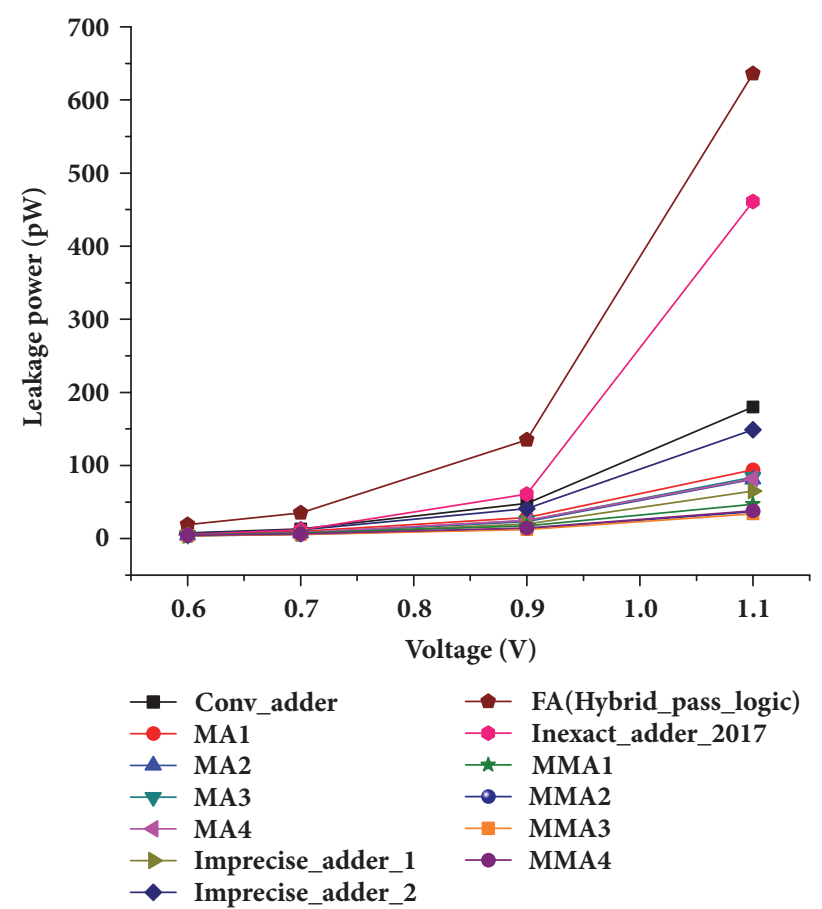

FIGURE 22: Effect on leakage power for existing and modified approximate FA due to variations in voltages.

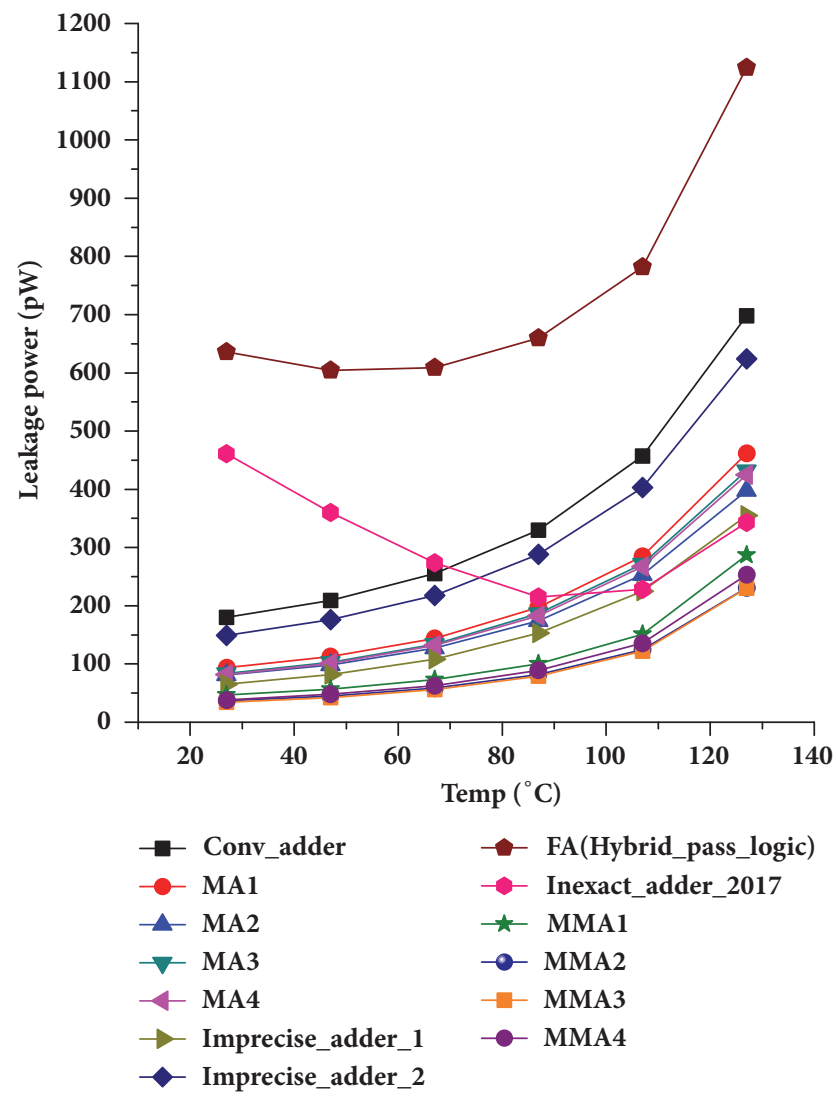

FIgURE 23: Effect on leakage power for existing and modified approximate adder due to variations in temperature.

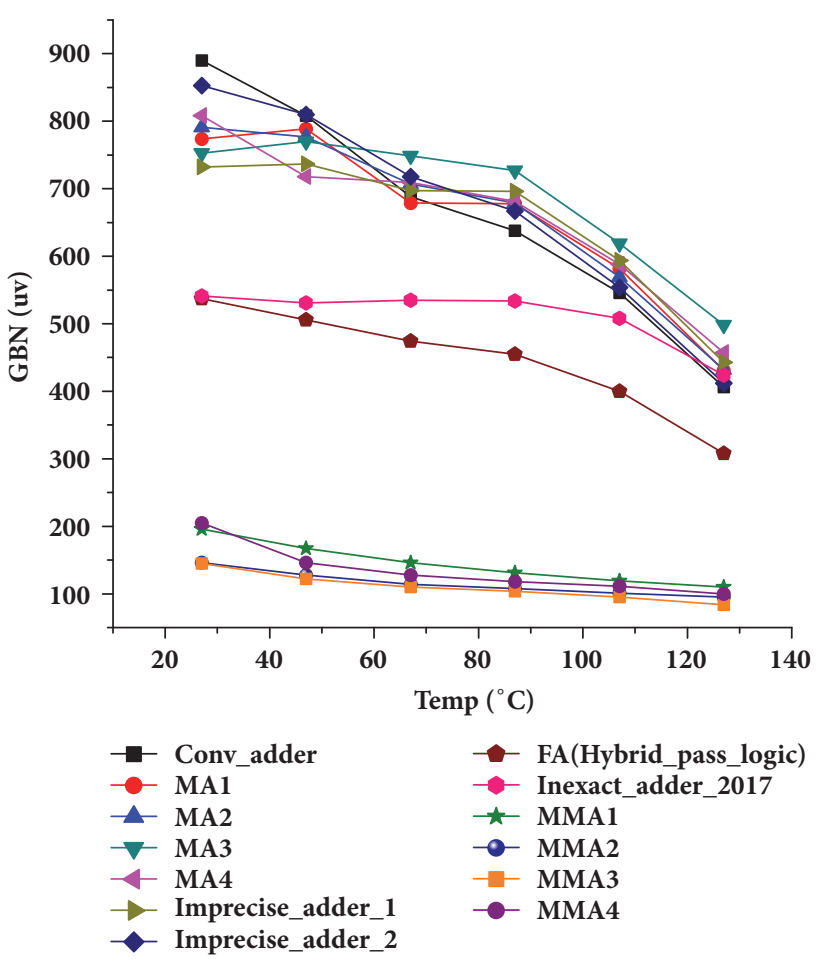

FIGURE 24: Effect of temperature variations for existing and modified approximate FA on GBN.

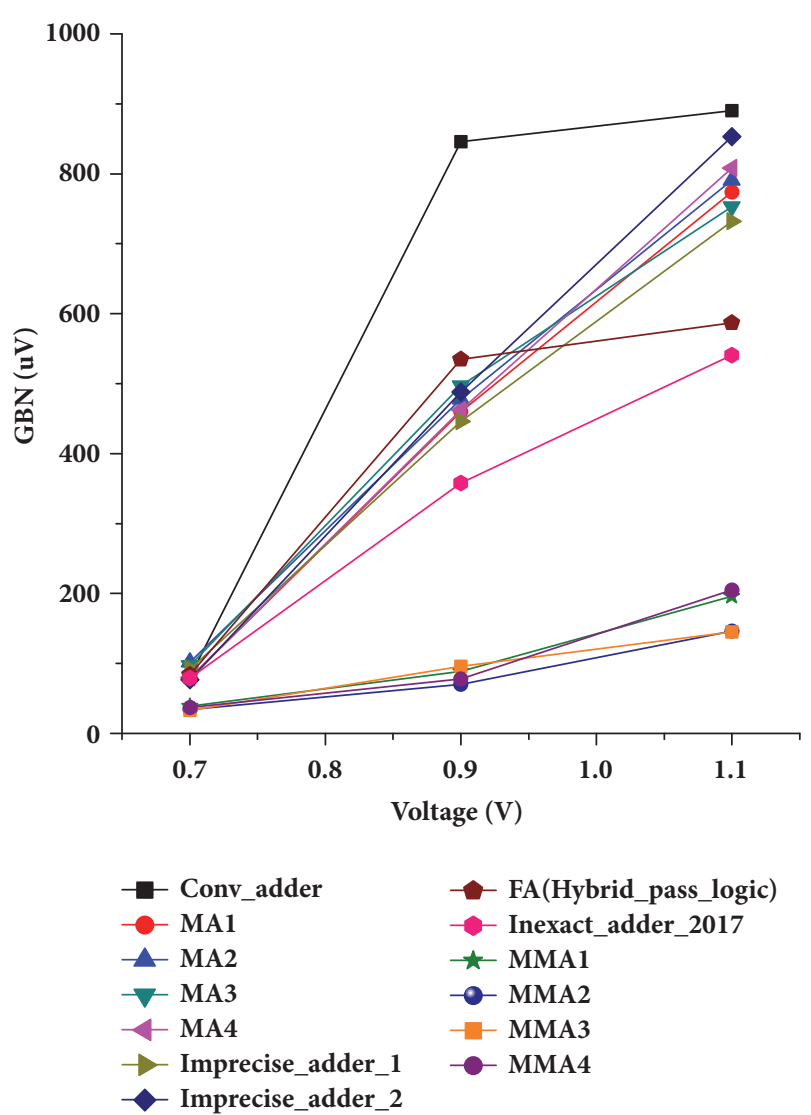

FIGURE 25: Effect of voltage variations for existing and modified approximate FA on GBN. 


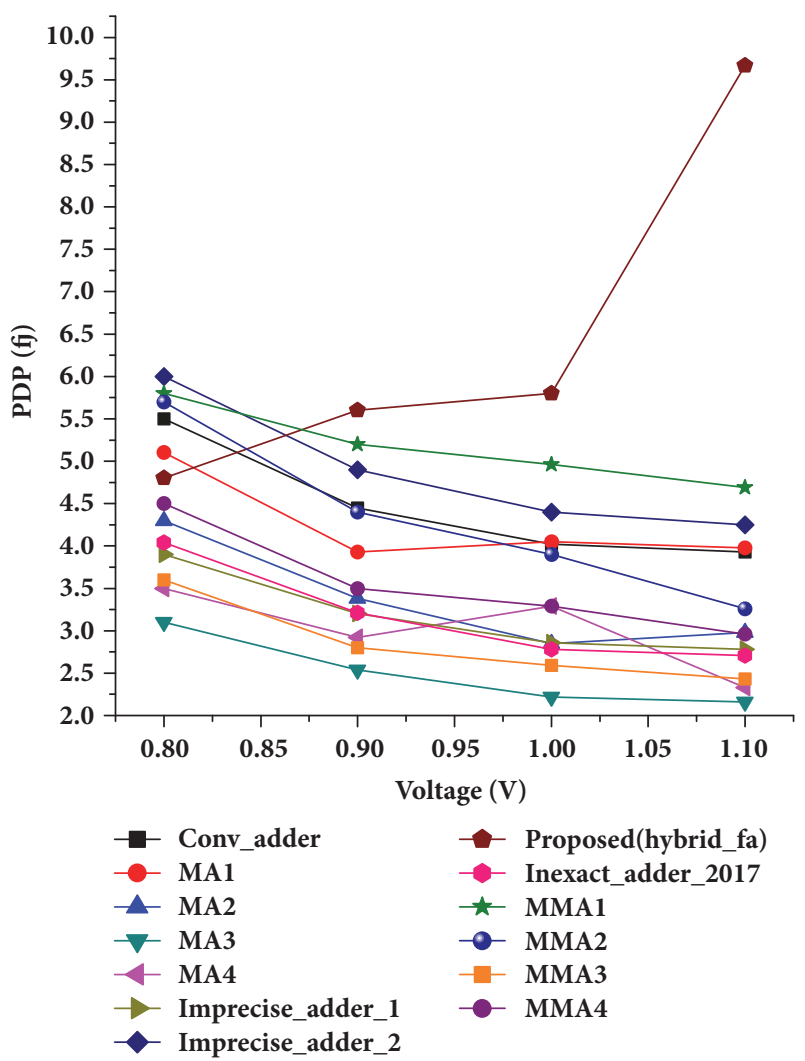

FIGURE 26: Effect of PDP with voltage variations for existing and modified approximate FA.

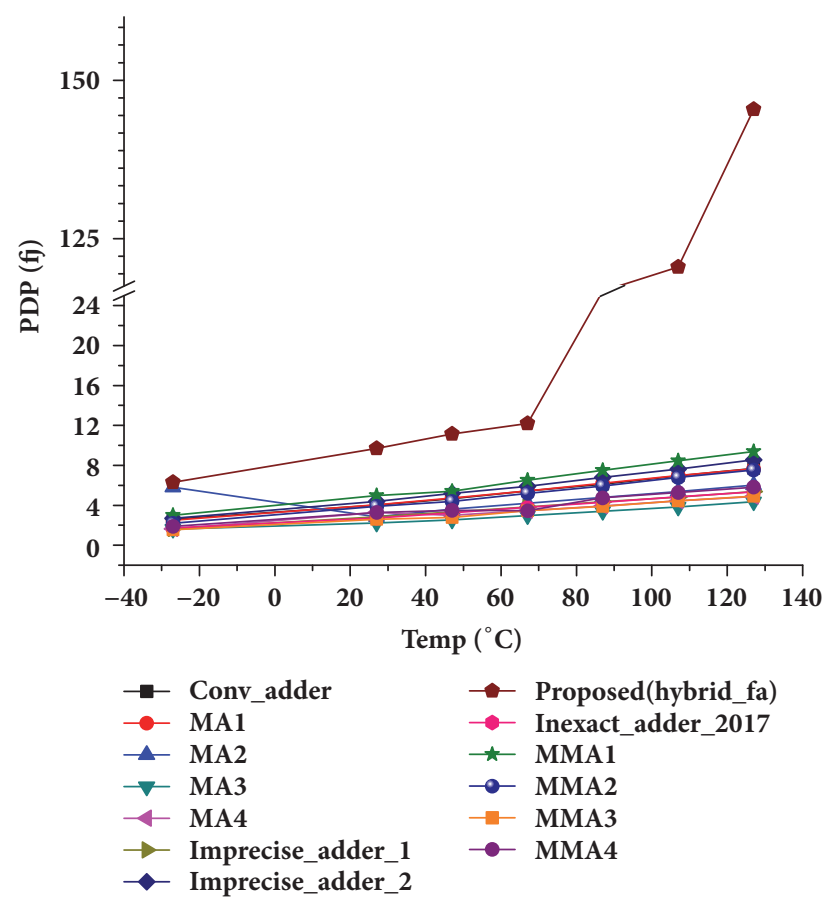

FIGURE 27: Effect of PDP with temperature variations for existing and modified approximate FA.

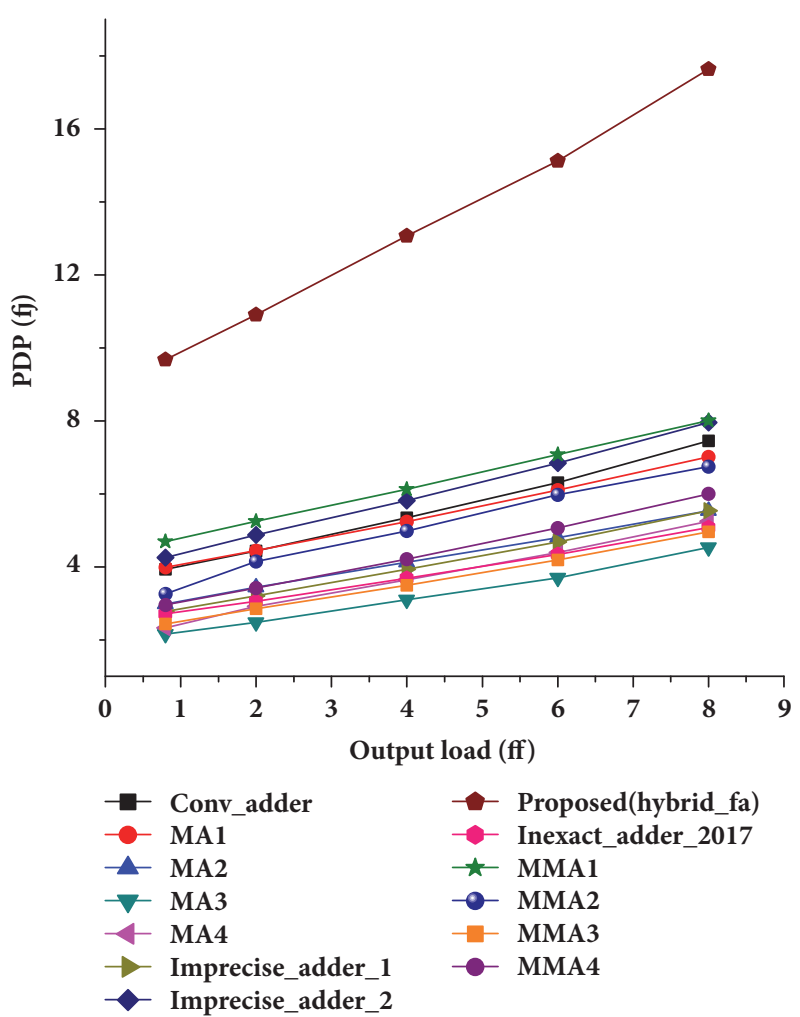

FIGURE 28: Effect of PDP with output load variations for existing and modified approximate FA.

picture. We have used two's complement for subtraction of $\mathrm{n}_{2}(\mathrm{i}, \mathrm{j})$ matrices which is shown in $(9)$.

$$
Q(i, j)=n_{1}(i, j)+\left[\text { two's complement of } n_{2}(i, j)\right]
$$

Addition operation in (9) is the binary addition of two matrices which is performed by using accurate and proposed approximate FA, respectively. $\mathrm{Q}(\mathrm{i}, \mathrm{j})$ matrices are converted into output image using MATLAB program. Figure 30 shows the resultant picture from $Q(i, j)$ matrices with absolute difference using all accurate adders.

Figure 31 shows the absolute difference between the frames using MMA1 adder (Figure 31(a)), MMA2 adder (Figure 31(b)), MMA3 adder (Figure 31(c)), and MMA4 adder (Figure 31(d)). As the number of approximate output increases, the output result tends to be inaccurate which is clearly visible in Figure 31.

Finally, the PSNR is measured for evaluating a quantitative metric to study the quality of the proposed approximate FA. The PSNR is measured using (10), where $M A X_{f}$ denotes the maximum signal value that exists in our original image and $e_{M S E}$ denotes mean-squared error.

$$
\begin{aligned}
P S N R & =10 \log _{10} \frac{M A X_{f}^{2}}{e_{M S E}} \\
e_{M S E} & =\frac{1}{m n} \sum_{i=1}^{m} \sum_{j=1}^{n}\left[I_{1}(i, j)-I_{2}(i, j)\right]^{2}
\end{aligned}
$$




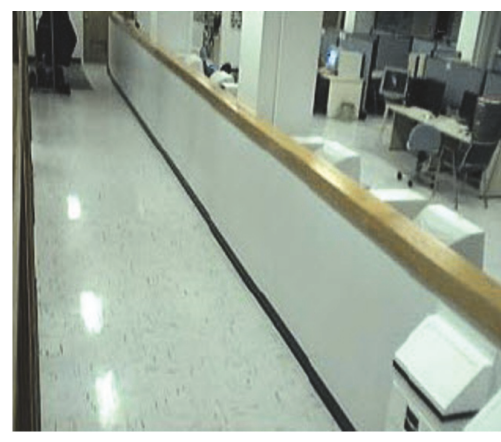

(a)

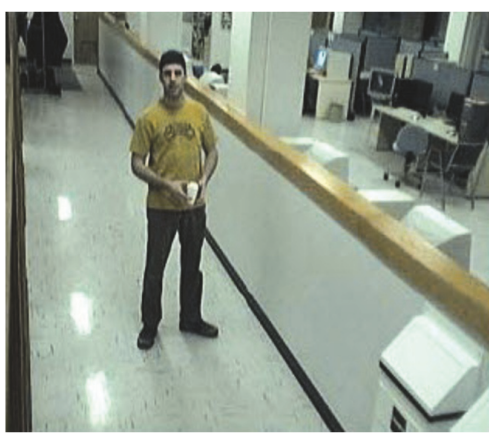

(b)

Figure 29: (a), (b) Reference images for motion detector.

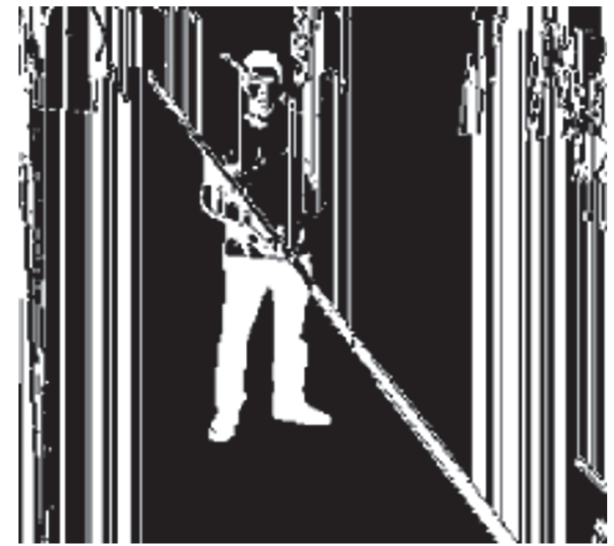

FIGURE 30: Resultant picture with absolute difference between two frames using all accurate adders.

TABLE 3: PSNR for the subtracted images by using an mmal, mma2, mma3 and mma4 proposed approximate FA.

\begin{tabular}{lcc}
\hline S.no. & Number of proposed approximate adder & PSNR \\
\hline 1. & MMA1 adder & 10.8 \\
\hline 2. & MMA2 adder & 20.2 \\
\hline 3. & MMA3 adder & 10.6 \\
\hline 4. & MMA4 adder & 6.65 \\
\hline
\end{tabular}

The $e_{M S E}$ is calculated using (11) where symbols $\mathrm{n}$ and $\mathrm{m}$ denote the number of rows and columns of a picture. Table 3 Shows the comparison of PSNR for the subtracted images by using proposed approximate adders. All the results in Table 3 are extracted through MATLAB program.

4.6. Comparison of Performance between Modified Approximate FA and Existing FAs. This section presents the performance comparison of the modified FA circuits with existing FA in terms of average power, delay, leakage power, PDP, and maximum GBN peaks. From the results in Table 2, it is observed that MAA3 adder has the best performance characteristics among all the modified adders, so we have compared the MAA3 adder with existing adders which have following advantages.

(1) The modified MAA3 adder has the least leakage power consumption $(34 \mu \mathrm{W})$ as compared to any other FA under consideration. The reduction in leakage power is achieved due to the reduction in potential at VGND node in sleep mode, which decreases the potential difference between source and drain of NMOS transistors in pull-down network of adder and hence gate leakage current reduces. This advantageous feature in one-bit adder makes the modified design a best choice for designing long chain arithmetic circuits at nanoscale VLSI technology.

(2) The modified MAA3 has delay metric as 316ps which is $12 \%$ more as compared to existing adder of the same type MA3. Increase in delay in MMA3 FA is due to the inclusion of sleep circuit.

(3) The GBN peaks of modified MMA3 adder is $145 \mu \mathrm{v}$ which is $80 \%$ less as compared to the same category (MA3) of existing adder. It is achieved because of the reduction in stored charge at VGND node during sleep mode. Also, NMOS transistor of proposed sleep circuit turns ON in linear region during sleep to active mode transition, which further reduces the voltage fluctuations and hence the GBN peaks. Reduction in GBN peaks makes this design a best choice for designing low leakage arithmetic circuits for portable electronic devices.

(4) MAA3 has the least average power dissipation among the modified adders and has almost equal average power dissipation when compared to existing adder MA3.

(5) MAA3 has $11 \%$ more PDP as compared MA3 adder, but still it has second rank if we compare MAA3 with all other existing adders under consideration as shown in Table 2.

Modified approximate FAs in this paper not only reduce GBN but also effectively reduce the leakage current using sleep circuit. Mostly, existing power gating techniques use multithreshold transistors to design sleep circuits, which increases complexity in designing of layout due to various extra processing steps. In all the modified approximate FA circuits, all the transistors have the same $V_{\text {th }}$ which makes layout design very simple. However, there is marginal increase in delay and PDP in modified circuit which is still in acceptable range. Reduction in leakage power and GBN 


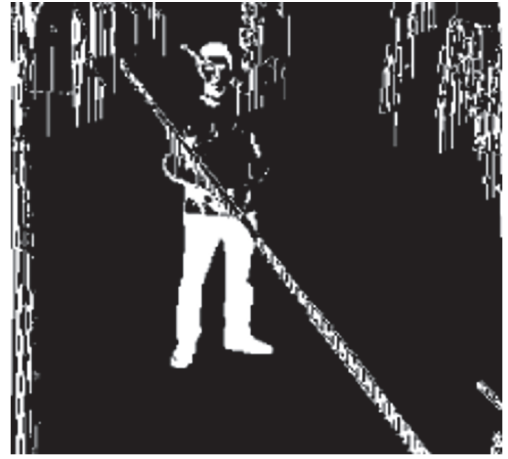

(a)
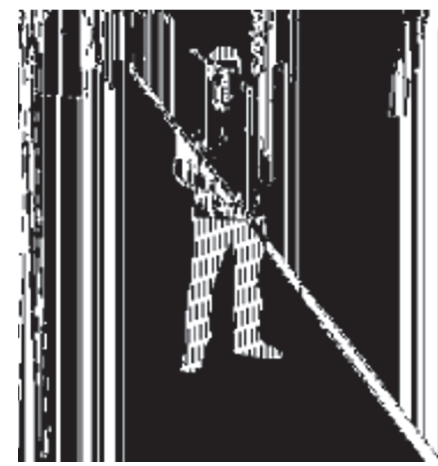

(b)

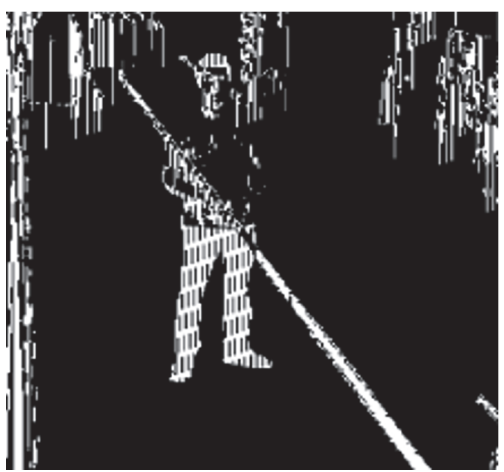

(c)

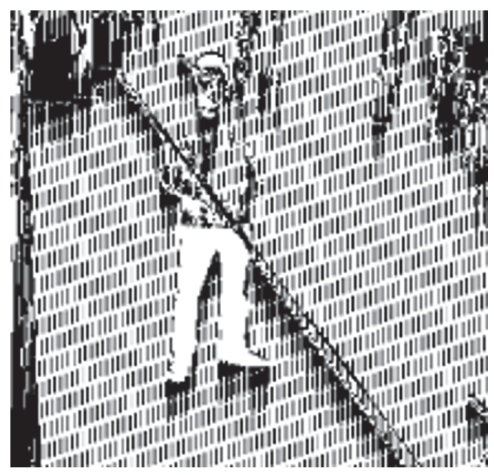

(d)

Figure 31: Absolute difference between the two frames by using (a) MMA1 adder, (b) MMA2 adder, (c) MMA3 adder, and (d) MMA4 adder.

has more weightage as compared to PDP and delay because modern portable electronic devices need low leakage circuit to improve the battery life in idle condition.

\section{Conclusion}

This paper presented four modified approximate adders which minimize the leakage power as well as controlling the GBN in transition mode. The modified approximate FAs are compared with other standard design approaches like MA1, MA2, MA3, MA4, imprecise adder, inexact adder, hybrid adder (FA), and conventional adder. The existing as well as modified FAs are simulated using post-layout netlist extracted from Cadence Virtuoso layout editor. Result shows that the modified design has the least leakage power dissipation and GBN in comparison to other adders. The modified designs offered $60 \%$ and $80 \%$ improvement in their leakage power and GBN, respectively, as compared to the best reported existing approximate FAs. The modified circuits of approximate adders are verified at various voltage and temperature ranges which confirm its robustness and reliability. Although modified design needed extra transistors, improvement in performance parameters makes this circuit a best choice for nanoscale VLSI design. It is concluded that the modified design is one of the best contenders for designing the long chain arithmetic circuit for low leakage power applications.

\section{Data Availability}

The data used to support the findings of this study are available from the corresponding author upon request.

\section{Conflicts of Interest}

The authors declare that they have no conflicts of interest.

\section{References}

[1] A. Chakraborty and S. N. Pradhan, "A new design of adder circuit for low power and high speed in sub-threshold region," International Journal of Electronics Letters, vol. 2, no. 1, pp. 17-29, 2014.

[2] G. Karimi and O. Sadeghi, "A novel adder cell for ultra low voltage, ultra low power networks in nanoscale VLSI circuits," IEICE Electronics Express, vol. 8, no. 7, pp. 478-483, 2011.

[3] R. Lorenzo and S. Chaudhury, "A new ultra low leakage and high speed technique for CMOS circuits," in Proceedings of the 2014 Students Conference on Engineering and Systems (SCES), pp. 1-5, Allahabad, India, May 2014.

[4] A. Yadav and S. Nakhate, "Low standby leakage 12T SRAM cell characterisation," International Journal of Electronics, vol. 103, no. 9, pp. 1446-1459, 2016.

[5] B. S. Deepaksubramanyan and A. Nunez, "Analysis of subthreshold leakage reduction in CMOS digital circuits," in Proceedings of the 2007 Joint 50th IEEE International Midwest 
Symposium on Circuits and Systems (MWSCAS) and the IEEE Northeast Workshop on Circuits and Systems (NEWCAS 2007), pp. 1400-1404, Montreal, QC, Canada, August 2007.

[6] H. V. Jayashree and K. Harsha, "Four BIT CMOS full adder in submicron technology with low leakage and Ground bounce noise reduction," in Proceedings of the 2012 International Conference on Devices, Circuits and Systems, ICDCS 2012, pp. 20-24, India, March 2012

[7] R. Singh and S. Akashe, "Design and analysis of low standby leakage current and reduce ground bounce noise of static CMOS 10 T full adder," African Journal of Computing \& ICT, vol. 7, no. 2, pp. 143-150, 2014.

[8] V. K. Sharma and M. Pattanaik, "A reliable ground bounce noise reduction technique for nanoscale CMOS circuits," International Journal of Electronics, vol. 102, no. 11, pp. 1852-1866, 2015.

[9] C. Goyal, J. S. Ubhi, and B. Raj, "Design of nano scale CMOS full adder with low leakage and ground bounce noise reduction," in Proceedings of the 2016 International Conference on Signal Processing and Communication (ICSC), pp. 380-385, Noida, India, December 2016.

[10] H. Jiao and V. Kursun, "Threshold voltage tuning for faster activation with lower noise in tri-mode MTCMOS circuits," IEEE Transactions on Very Large Scale Integration (VLSI) Systems, vol. 20, no. 4, pp. 741-745, 2012.

[11] R. Singh, "Akashe, Modeling and analysis of low power 10T full adder with reduced ground bounce noise," Journal of Circuits. Systems \& Computers, vol. 23, no. 1, p. 14, 2014.

[12] S. Kim, S. V. Kosonocky, D. R. Knebel, K. Stawiasz, and M. C. Papaefthymiou, "A multi-mode power gating structure for low-voltage deep-submicron CMOS ICs," IEEE Transactions on Circuits and Systems II: Express Briefs, vol. 54, no. 7, pp. 586-590, 2007.

[13] P. Sreenivasulu, K. Srinivasa Rao, and A. Vinaya Babu, "Ground bouncing noise reduction in combinational MTCMOS circuits," in Proceedings of the 2014 International Conference on Communication and Network Technologies, ICCNT 2014, pp. 270-275, India, December 2014.

[14] H. Jiang, J. Han, and F. Lombardi, "A comparative review and evaluation of approximate adder," in Proceedings of the 25th edition on Great Lakes Symposium on VLSI, pp. 343-348, 2015.

[15] Z. Yang, J. Han, and F. Lombardi, "Transmission gate-based approximate adders for inexact computing," in Proceedings of the IEEE/ACM International Symposium on Nanoscale Architectures, NANOARCH 2015, pp. 145-150, USA, July 2015.

[16] D. Celia, V. Vasudevan, and N. Chandrachoodan, "Optimizing power- accuracy trade-off in approximate adders," in Proceedings of the Design, Automation Test in Europe Conference \& Exhibition (DATE), pp. 1488-1491, 2018.

[17] H. A. Almurib, T. Nandha Kumar, and F. Lombardi, "Approximate DCT image compression using inexact computing," Institute of Electrical and Electronics Engineers. Transactions on Computers, vol. 67, no. 2, pp. 149-159, 2018.

[18] I. Alam and K. T. Lau, "Approximate adder for low-power computations," International Journal of Electronics Letters, vol. 1724, pp. 1-8, 2016.

[19] V. Gupta, D. Mohapatra, A. Raghunathan, and K. Roy, "Lowpower digital signal processing using approximate adders," IEEE Transactions on Computer-Aided Design of Integrated Circuits and Systems, vol. 32, no. 1, pp. 124-137, 2013.

[20] Y. S. Mehrabani, R. F. Mirzaee, Z. Zareei, and S. M. Daryabari, "A Novel High-Speed, Low-Power CNTFET-Based Inexact
Full Adder Cell for Image Processing Application of Motion Detector," Journal of Circuits, Systems and Computers, vol. 26, no. 5, 2017.

[21] M. C. Parameshwara and H. C. Srinivasaiah, "Low-Power Hybrid 1-Bit Full-Adder Circuit for Energy Efficient Arithmetic Applications," Journal of Circuits, Systems and Computers, vol. 26, no. 01, p. 1750014, 2017.

[22] R. Bhanuprakash, M. Pattanaik, S. S. Rajput, and K. Mazumdar, "Analysis and reduction of ground bounce noise and leakage current during mode transition of stacking power gating logic circuits," in Proceedings of the 2009 IEEE Region 10 Conference, TENCON 2009, Singapore, November 2009. 


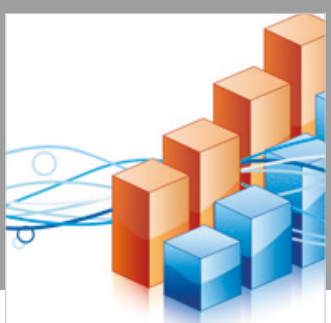

Advances in

Operations Research

\section{-n-m}
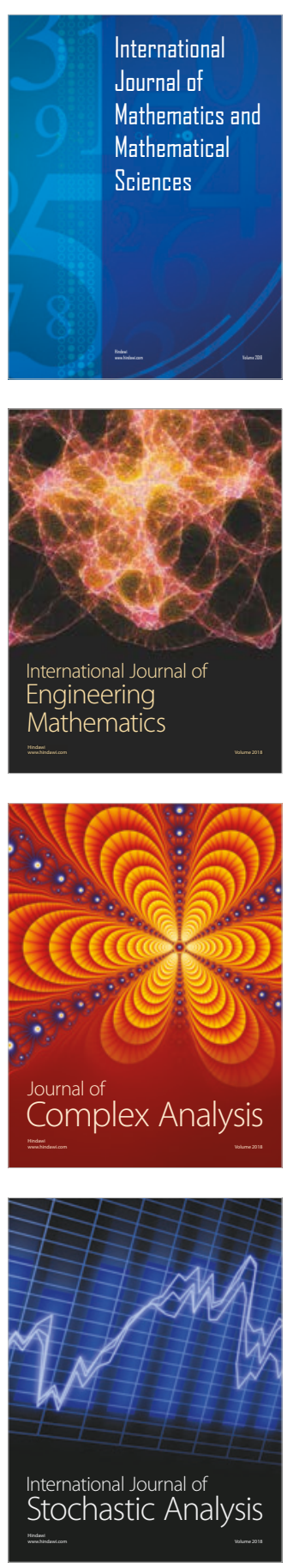
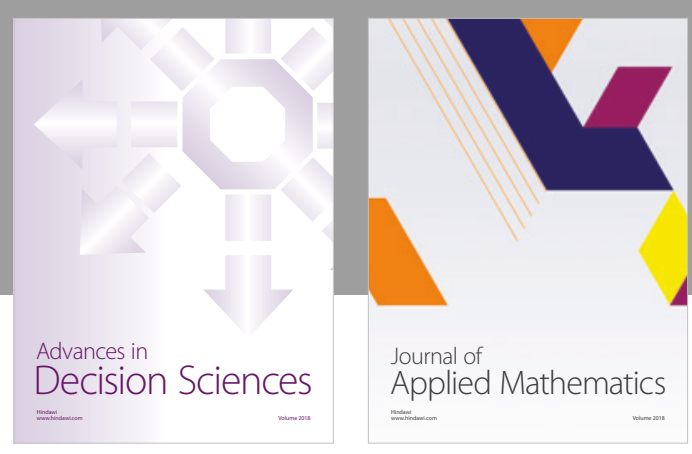

Journal of

Applied Mathematics
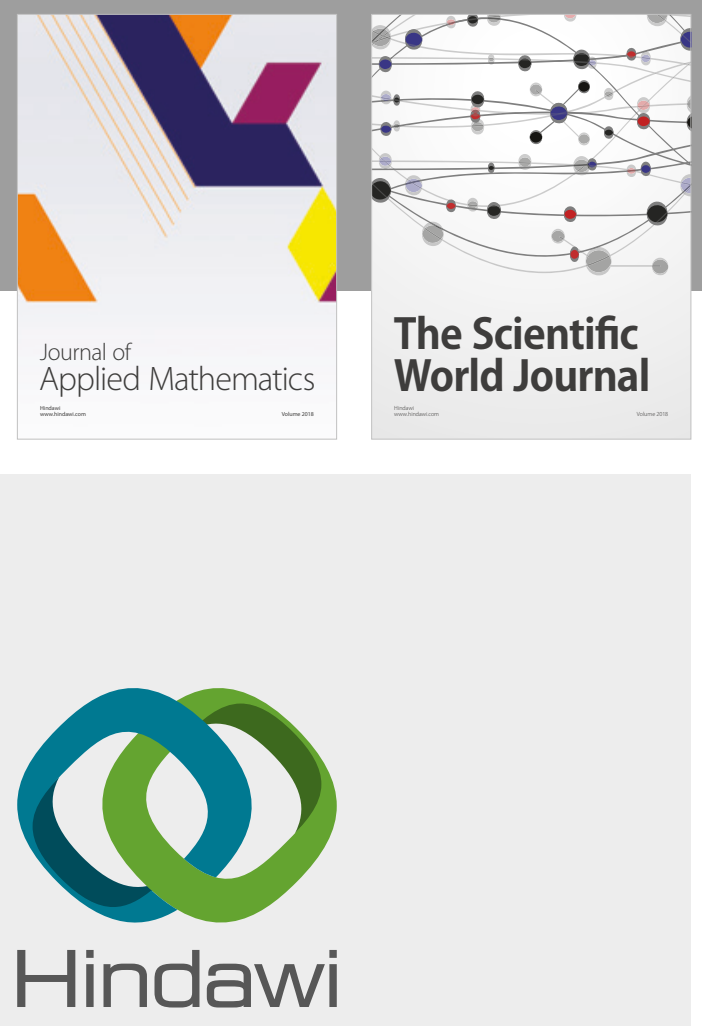

Submit your manuscripts at

www.hindawi.com

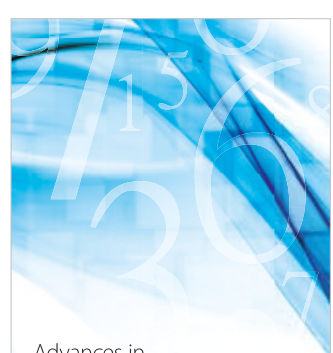

Advances in
Numerical Analysis
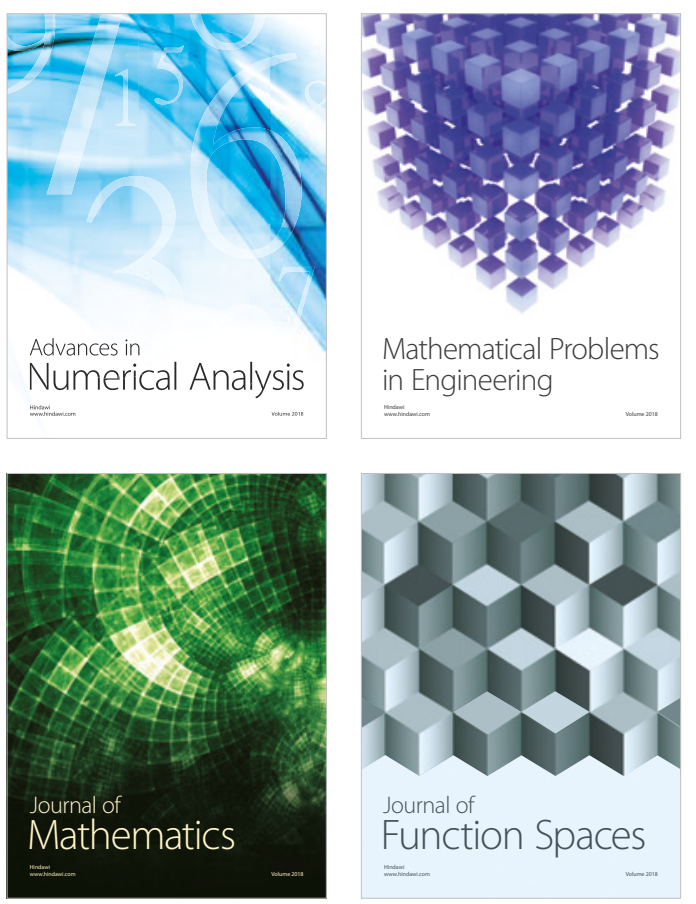

Mathematical Problems in Engineering

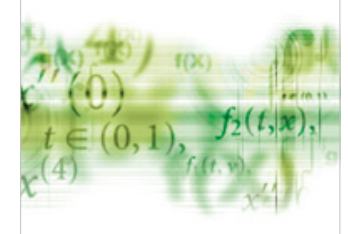

International Journal of

Differential Equations

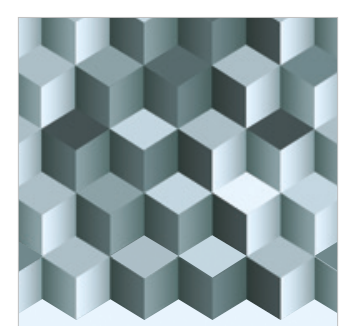

Journal of

Function Spaces

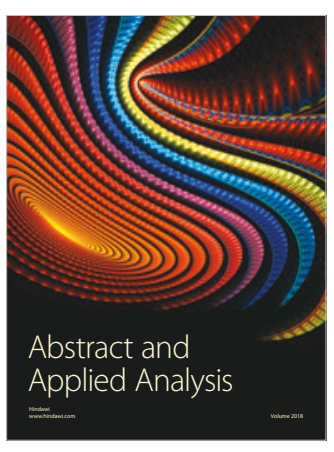

The Scientific

World Journal

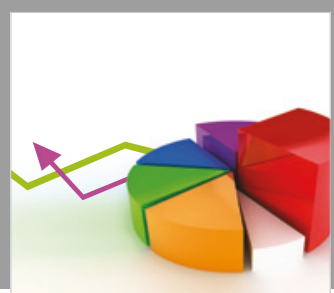

Journal of

Probability and Statistics
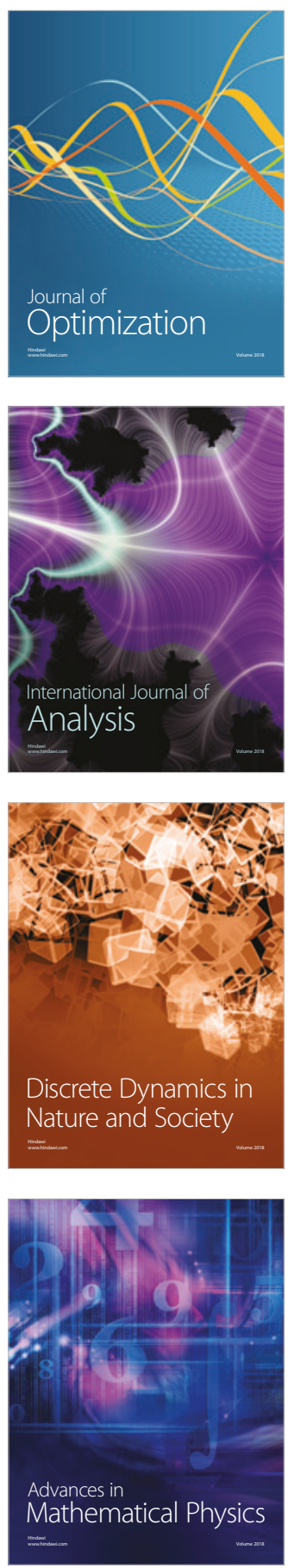